Dear author,

Please note that changes made in the online proofing system will be added to the article before publication but are not reflected in this PDF.

We also ask that this file not be used for submitting corrections. 


\section{Advanced technologies for satellite navigation and geodesy}

\section{G. Giorgi ${ }^{\text {a }}$, T.D. Schmidt ${ }^{\text {a }}$, C. Trainotti ${ }^{\text {a }}$, R. Mata-Calvo ${ }^{\text {a }}$, C. Fuchs ${ }^{\text {a }}$, M.M. Hoque ${ }^{\text {a }}$, J. Berdermann ${ }^{\text {a }}$, J. Furthner ${ }^{\text {a }}$, C. Günther ${ }^{\mathrm{a}}$, T. Schuldt ${ }^{\mathrm{b}}$, J. Sanjuan ${ }^{\mathrm{b}}$, M. Gohlke ${ }^{\mathrm{b}}$, M. Oswald ${ }^{\mathrm{b}, \mathrm{c}}$, C. Braxmaier ${ }^{\mathrm{b}, \mathrm{c}}$, K. Balidakis ${ }^{\mathrm{d}}$, G. Dick ${ }^{\mathrm{d}}$, F. Flechtner ${ }^{\mathrm{d}}$, M. Ge ${ }^{\mathrm{d}}$, S. Glaser ${ }^{d}$, R. König ${ }^{d}$, G. Michalak ${ }^{d}$, M. Murböck ${ }^{d}$, M. Semmling ${ }^{d}$, H. Schuh ${ }^{d}$ \\ ${ }^{a}$ Institut für Kommunikation und Navigation, Deutsches Zentrum für Luft- und Raumfahrt e.V., Oberpfaffenhofen, Germany \\ ${ }^{\mathrm{b}}$ Institut für Raumfahrtsysteme, System Enabling Technologies, Deutsches Zentrum für Luft- und Raumfahrt e. V., Bremen, Germany \\ ${ }^{\mathrm{c}}$ Universität Bremen, Zentrum für Angewandte Raumfahrttechnologie und Mikrogravitation (ZARM), Bremen, Germany \\ ${ }^{\mathrm{d}}$ GFZ German Research Center for Geosciences, Potsdam, Germany}

Received 22 March 2019; received in revised form 31 May 2019; accepted 9 June 2019

\section{Abstract}

This manuscript reviews recent progress in optical frequency references and optical communication systems and discusses their utilizations in global satellite navigation systems and satellite geodesy. Lasers stabilized with optical cavities or spectroscopy of molecular iodine are analyzed, and a hybrid architecture is proposed to combine both forms of stabilization with the aim of achieving a target frequency stability of $10^{-15}[\mathrm{~s} / \mathrm{s}]$ over a wide range of sampling intervals.

The synchronization between two optical frequency references in real-time is realized by means of time and frequency transfer on optical carriers. The technologies enabling coherent optical links are reviewed, and the development of an optical communication system for synchronization, ranging and data communication in space is described. An infrastructure exploiting the capabilities of both optical technologies for the realization of a modernized constellation of navigation satellites emitting highly synchronized signals is reviewed. Such infrastructure, named Kepler system, improves satellite navigation in terms intra-system synchronization, orbit determination accuracy, as well as system monitoring and integrity. The potential impact on geodetic key parameters is addressed.

(C) 2019 COSPAR. Published by Elsevier Ltd. All rights reserved.

Keywords: Optical frequency references; Cavity-stabilized lasers; Iodine clock; Optical inter-satellite links; Inter satellite ranging; GNSS; Navigation; Geodesy; Terrestrial Reference Frames; Precise Orbit Determination; Atmosphere sensing; Kepler

Abbreviations: ACES, Atomic Clock Ensemble in Space; AOM, Acusto-Optical Modulator; ARTEMIS, Advanced Relay and TEchnology MISsion Satellite; CCD, Charge-Coupled Device; CPA, Coarse Pointing Assembly; CTE, Coefficient of Thermal Expansion; DLL, Delay-Locked-Loop; DORIS, Doppler Orbitography and Radiopositioning Integrated by Satellite; EBB, Elegant BreadBoard; ECDL, External Cavity Diode Lasers; EDRS, European Data Relay System; EOM, Electro-Optical Modulator; FSR, Free Spectral Range; GNSS, Global Navigation Satellite System; GPS, Global Positioning System; GRACE, Gravity Recovery and Climate Experiment; GRACE-FO, GRACE Follow-On; IEM, Implicit Ensemble Mean; ISL, Inter-Satellite Links; ITRF, International Terrestrial Reference Frame; LCT, Laser Communication Terminal; LEO, Low Earth Orbit; LISA, Laser Interferometer Space Antenna; LTT, Laser Time Transfer; MEO, Medium Earth Orbit; NGGM, Next Generation Gravity Mission; OADEV, Overlapping Allan Deviation; OISL, Optical Inter-Satellite Links; PDH, Pound-Drever-Hall; POD, Precise Orbit Determination; PPLN, Periodically Poled Lithium Niobate; RAM, Residual Amplitude Modulation; SILEX, Semiconductor laser Intersatellite Link EXperiment; SISRE, Signal-In-Space Range Error; SLR, Satellite Laser Ranging; SNR, Signal-to-Noise Ratio; T2L2, Time Transfer by Laser Link; TRF, Terrestrial Reference Frame; ULE, Ultra-Low Expansion; UTC, Coordinated Universal Time; VLBI, Very Long Baseline Interferometry

E-mail address: gabriele.giorgi@dlr.de (G. Giorgi). 


\section{Introduction}

During the last decades, two key technologies have emerged that push the boundaries of time keeping and space communications: optical frequency references and inter-satellite laser links. This manuscript reviews these optical technologies for advancing satellite navigation, geodesy and time-metrology, and discusses recent activities on design, development, testing and exploitation of key optical components. These activities include the engineering of cavity- and iodine-based frequency references, and their characterization in terms of frequency stability; the realization of a hybrid clock formed by an optical cavity and an absolute iodine frequency reference; the design and test of optical terminals and transmitters/receivers for coherent inter-satellite laser links; and the system architecture combining each of these technologies to establish a network of space-based synchronized references capable of providing highly accurate timing and positioning services.

Satellite navigation, satellite geodesy and time metrology deal with two aspects of time: generation and dissemination. Quantitatively measuring the flow of time consists in defining a time scale, based on the elapsed time or repetition frequency of periodically-recurring events, such as the yearly motion of the Earth around the sun, the 28-day motion of the moon around the Earth, or the swing of a pendulum. Nowadays, the most stable frequency standards are based upon atomic clocks, which lock to an atomic or molecular electronic transition in the microwave regime. Example of such references are Cesium, Hydrogen and Rubidium atomic clocks, whose relative stabilities in terms of Allan deviation range from $10^{-9}$ to $10^{-13}[\mathrm{~s} / \mathrm{s}]$ at $1 \mathrm{~s}$, and from $10^{-14}$ to $10^{-15}[\mathrm{~s} / \mathrm{s}]$ at $10^{5} \mathrm{~s}$. Optical frequency references are capable of providing superior frequency stability, outperforming the current standards operating in the microwave frequency range. Optical ion and lattice clocks have demonstrated frequency stabilities below $10^{-18}[\mathrm{~s} / \mathrm{s}]$ for long sample intervals (Bloom et al., 2014; Ushijima et al., 2015). Less complex setups based on Doppler-free spectroscopy show frequency stabilities at the $10^{-15}[\mathrm{~s} / \mathrm{s}]$ level for sample intervals between $1 \mathrm{~s}$ and $10^{3} \mathrm{~s}$ (Schuldt et al., 2017), already taking into account design aspects relevant for space-based utilizations such as compactness and rigidity. Furthermore, laboratory tests of optical frequency references based on laser interferometry (optical cavities) show short-term frequency stabilities below $10^{-15}[\mathrm{~s} / \mathrm{s}]$ for sample intervals up to $10 \mathrm{~s}$ (see e.g. Jiang et al., 2011).

The output of two frequency references can be remotely compared via two-way time and transfer techniques. Communication systems based on Nd:YAG lasers, already space-qualified and operative for commercial satellite data relay applications, represent the state of the art for highrate free-space communications (Surof et al., 2017; Schmidt and Fuchs, 2017). Early ground-to-space tests of time transfer via non-coherent optical links have already demonstrated accuracies in the range of hundreds picoseconds. Examples of such tests are the Laser Time Transfer technology validated at 300 ps-level on Beidou satellites (Meng et al., 2012), the Time Transfer by Laser Link (T2L2), which demonstrated time transfer between a number of International Laser Ranging Service (ILRS) stations and the Jason-2 satellite below the 100 ps-level (Exertier et al., 2014), and an upcoming (2019) second iteration of the T2L2 named European Laser Timing that will enable time transfer with the stable atomic clocks part of the Atomic Clocks Ensemble in Space (ACES) experiment (Schreiber et al., 2010). All these methods use noncoherent links. By exploiting laser-based coherent links, a network of two or more frequency references synchronized at sub-ps level can be established. A set of synchronized optical frequency references characterized by short-term frequency stabilities at the $10^{-15}[\mathrm{~s} / \mathrm{s}]$ level carried by Earth-orbiting satellites offers the potential to greatly enhance the performance of global navigation systems and to support precise satellite geodesy applications (Günther, submitted for publucation). A network of satellites interconnected via bi-directional free-space optical links chained to the local frequency-stabilized laser oscillators creates a tightly synchronized time reference that can be realized via a space-based clock ensemble. Such architecture also enables a better separation of uncertainties in the spatial and time domains, while providing high rate communications and absolute ranging with sub-mm level accuracy, which in turn enhances orbit determination.

These elements are the basis of the Kepler proposal for a future satellite navigation system, which is based on an extremely stable and robust time scale. In this concept Medium Earth Orbit (MEO) navigation satellites equipped with cavity-stabilized lasers and inter-connected via coherent laser links shall enable the broadcast of navigation signals synchronized to a level far superior to modern GNSSs. The essential elements of the concept, presented in (Günther, submitted for publication, 2018a), are reviewed in Section 4 for the sake of clarity.

Other than improving satellite-based navigation, globally available synchronized signals have a direct impact on a myriad of applications. Notable examples are time distribution across continents, the provision of terrestrial reference frames and Earth rotation parameters, enhanced gravity field retrieval (especially for long wavelengths) thanks to an improved positioning service for dedicated Low Earth Orbit (LEO) probes, or the recovery of atmospheric zenith delays and gradient vector components via the higher accuracy of MEO orbit estimates.

This manuscript intends to provide an up-to-date overview on optical timing and communication technologies and address their utilization in satellite-based navigation and geodesy. The technological aspects of optical frequency references and laser communication and ranging systems are described in Sections 2 and 3, respectively, with focus on design and overall expected performance. The exploitation of the two key technologies in future global satellite navigation system architectures is analyzed in Section 4 , in which a system-engineering overview is provided. 
Section 4 also reviews potential enhancements in satellite geodesy applications stemming from the capabilities offered by the envisioned GNSS architecture.

\section{Optical frequency references and clocks}

Optical frequency references in the form of stabilized lasers are the most stable references that have been developed so far. Over the last decades, optical clock technologies evolved, recently demonstrating frequency stabilities at the $10^{-18}[\mathrm{~s} / \mathrm{s}]$ level for sample intervals of a few thousand seconds (see e.g. Bloom et al., 2014; Ushijima et al., 2015; Ludlow et al., 2015), surpassing microwave clocks' performance by several orders of magnitude. Different methods for laser frequency stabilization are conceivable, including stabilization to an optical cavity or to an atomic or molecular transition. While cavity-stabilized systems offer high short-term frequency stability (for sample intervals between milliseconds and a few seconds), atoms and molecules offer high long-term frequency stability (for sample intervals longer than $100 \mathrm{~s}$ ) and the provision of an absolute frequency reference. A combination of both systems in a single laser system - in the following referred to as hybrid clock - would take advantage of these properties at different time scales (Musha et al., 2000). For operation as a clock, an additional frequency comb is necessary, transferring the optical frequencies to the microwave regime, where counters are available. Current versions of frequency combs have already successfully been operated on sounding rockets (Lezius et al., 2016).

Ultimate frequency stability is shown using ion and lattice clock technologies in complex laboratory setups (Bloom et al., 2014; Ushijima et al., 2015), not yet available for space utilizations. However, optical frequency references based on Doppler-free spectroscopy and optical cavities can be realized in space-compatible compact and ruggedized setups. At $1064 \mathrm{~nm}$, space-proven lasers and (electro-) optic components are available. This wavelength is used in missions such as LISA (Laser Interferometer Space Antenna) Pathfinder (Armano et al., 2016) and GRACE-FO (Gravity Recovery and Climate Experiment Follow-On, Sheard et al., 2012) and it is the operating wavelength of the Laser Communication Terminals (LCTs, see Section 3). By second harmonic generation, Dopplerfree spectroscopy of molecular iodine near $532 \mathrm{~nm}$ can be implemented.

We report on current and future work on optical frequency references based on molecular iodine and on optical cavities, and on the implementation of a stable hybrid clock with targeted frequency stability at the $10^{-15}[\mathrm{~s} / \mathrm{s}]$ level for sample intervals between a few milliseconds and $10.000 \mathrm{~s}$.

\subsection{Iodine-based absolute frequency references}

Frequency references based on Doppler-free spectroscopy of molecular iodine at a wavelength near
$532 \mathrm{~nm}$ have been commonly used in laboratories for many years. They use either frequency modulation spectroscopy (Bjorklund, 1980; Arie and Byer, 1993) or modulation transfer spectroscopy (MTS) (Shirley, 1982; Ye at al., 2001; Zang at al., 2007), and are also developed in compact setups (Hong et al., 2001; Nyholm et al., 2003) and for space applications (Argence et al., 2010; Acef et al., 2010; Schuldt et al., 2017; Döringshoff et al., 2017; Schkolnik et al., 2017; Döringshoff et al., 2019).

In a collaboration between the German Aerospace Center (DLR Institute of Space Systems, Bremen), the University Bremen (Center of Applied Space Technology and Microgravity, ZARM) the Humboldt-University Berlin and Airbus $\mathrm{GmbH}$ (Friedrichshafen), two compact iodine-based frequency references on elegant breadboard (EBB) and engineering model (EM) level, respectively, were realized during the last years (Schuldt et al., 2017; Döringshoff et al., 2017). The corresponding spectroscopy units, which include the optical components for modulation transfer spectroscopy, use a baseplate made of glass material in combination with a dedicated easy-to-handle assembly-integration technology where the optical components are bonded to the baseplate using a space-qualified two-component epoxy (Ressel et al., 2010). This ensures high pointing stability of the two counter-propagating laser beams in the iodine cell needed for long-term frequency stability and the robustness required for space operations. The light source is a $1064 \mathrm{~nm}$ solid-state Nd:YAG laser which is frequency doubled to $532 \mathrm{~nm}$ using periodically poled lithium niobate (PPLN) waveguide technology.

Fig. 1, left, shows a photograph of the EM spectroscopy setup using a $38 \times 18 \times 4 \mathrm{~cm}^{3}$ fused silica baseplate and a compact gas cell, designed for nine-pass operation of pump and probe beam. The EBB was characterized in a beat measurement with an ultra-low expansion (ULE) cavity setup where noise levels of $6 \times 10^{-15}[\mathrm{~s} / \mathrm{s}]$ for $1 \mathrm{~s}$ sample intervals and below $3 \times 10^{-15}$ for sample intervals between $100 \mathrm{~s}$ and $1.000 \mathrm{~s}$ have been measured (Schuldt et al., 2017). The associated Allan Deviation is shown in Fig. 1, right. For sample intervals longer than $100 \mathrm{~s}$, the measurement is limited by the ULE cavity. The frequency stability is comparable to the active hydrogen maser as currently integrated for the ACES mission (Goujon et al., 2010) and represents the best results reported for an iodine-based system (Schuldt et al., 2017). The EM spectroscopy unit was subjected to thermal cycling from $-20^{\circ} \mathrm{C}$ to $+60{ }^{\circ} \mathrm{C}$ and vibrational loads with sine vibration up to $30 \mathrm{~g}$ and random vibration up to $25.1 \mathrm{~g}$. The frequency stability was measured before and after the tests, and no degradation was observed. These measurements showed an absolute frequency offset between EBB and EM setups below $1.5 \mathrm{kHz}$ with reproducibility below $250 \mathrm{~Hz}$ (Döringshoff et al., 2017).

In a recent activity, a very compact spectroscopy setup has been implemented using micro-integrated external cavity diode lasers (ECDLs) as light source (Schkolnik et al., 2017). It was successfully flown on a sounding rocket 

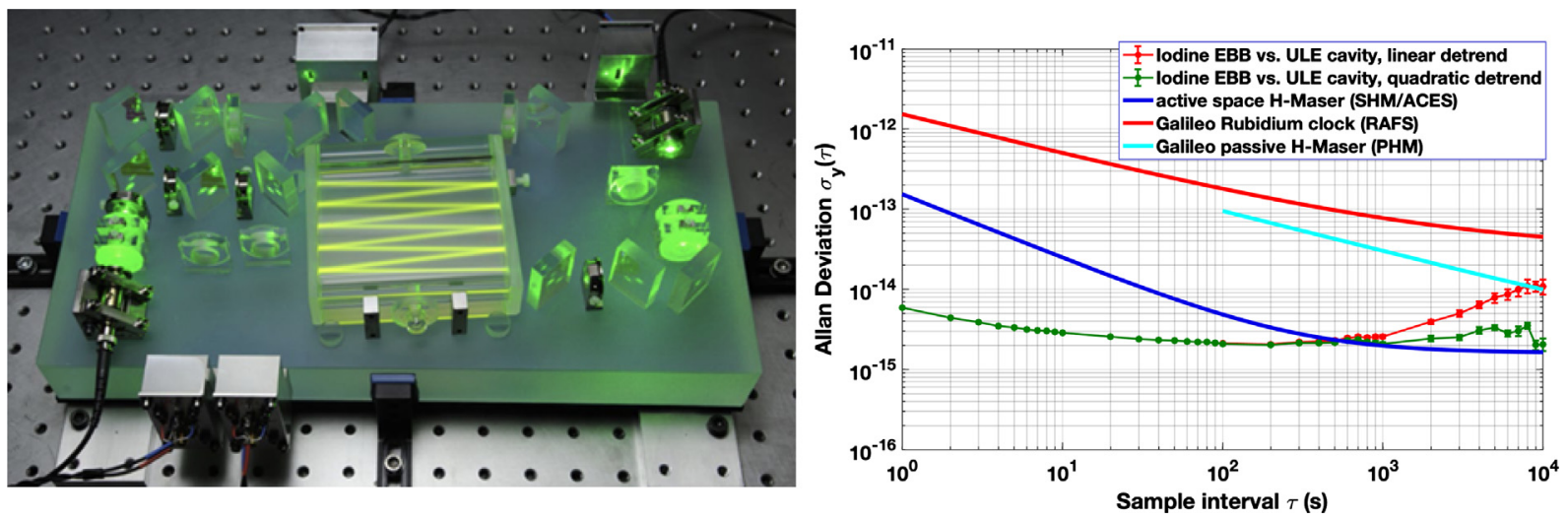

Fig. 1. Left: Photograph of the iodine spectroscopy unit on engineering model level (Döringshoff et al., 2017); Right: Frequency stability of the iodine EBB setup when compared to a ULE cavity setup (Schuldt et al., 2017), given in Allan Deviation. Also shown are the stabilities of the current space microwave clocks as flown on Galileo and foreseen for ACES.

mission, launched in May 2018 (Döringshoff et al., 2019). For future applications in satellite navigation, a new integrated setup with beam preparation and spectroscopy unit within one physical housing is currently being developed (Schuldt et al., 2018).

\subsection{Cavity-based frequency references}

Optical cavities have been used as frequency references since the 1960s and they have become essential in many fields of physics: gravitational waves observatories, precision spectroscopy, tests of Lorentz invariance, Earth gravity field monitoring, optical atomic clocks, laser metrology, etc. Optical cavities provide the frequency discriminator (the derivative of the cavity linewidth) needed to stabilize a laser by means of a servo loop. The discriminator is usually generated using the well-known Pound-Drever-Hall (PDH) method (Drever et al., 1983). In this manner, the length stability of the cavity is transferred to the laser frequency, which implies that extremely stable materials in very benign environments are required.

Optical cavities are a mature technology with many designs and configurations available for different purposes depending on requirements, frequencies of interest and environmental conditions. Laboratory set-ups operating at room temperatures using $\sim 10 \mathrm{~cm}$ long ULE glass spacers with fused silica mirrors reach thermal noise limits of $10^{-15} \mathrm{~s} / \mathrm{s}$ ] for sample intervals of a few seconds (Notcutt et al., 2005; Webster et al., 2008; Ludlow et al., 2007). In order to further reduce the thermal noise limit to the $10^{-}$ 16 [s/s] level and below, different approaches are followed including cryogenic temperatures using single crystal cavities (Kessler et al., 2012; Matei et al., 2016; Matei et al., 2017) and very long ULE cavities (near $50 \mathrm{~cm}$ ) at room temperature (Häfner et al., 2015; Jiang et al., 2011; Jin et al., 2018; Nicholson et al., 2012). Neither of these solutions is very attractive for space missions, due to size and mass constraints, sensitivity to misalignments or cryogenic temperature management. Promising new coatings have been reported, which exhibit an order of magnitude reduction in coating loss angle (Cole et al., 2013) and will allow reducing the thermal noise limit without cryogenic temperatures or very long cavities.

There have been considerable efforts on developing portable cavity-stabilized systems (Leibrandt et al., 2011a, 2011b; Vogt et al., 2011; Chen et al., 2014) and on increasing the technology readiness level for space applications (Argence et al., 2012, Swierad et al., 2016). For the latter, frequency stability of $5 \cdot 10^{-16}[\mathrm{~s} / \mathrm{s}]$ at $1 \mathrm{~s}$ and $2 \cdot 10^{-15}[\mathrm{~s} / \mathrm{s}]$ between 1 and $1.000 \mathrm{~s}$ have been demonstrated. Finally, the first cavity-stabilized laser in space is part of the laser ranging interferometer on board the GRACE-FO mission and has been successfully flying since May 22nd 2018 (Sheard et al., 2012; Thompson et al., 2011).

In order to reach the fundamental thermal noise limit one needs to take care of several technical noise sources. Some of them affect the actual length stability of the cavity, while the others are related to the error in signal generation, servo loops, etc. The former are due to temperature fluctuations that expand/contract the spacer due to nonzero coefficient of thermal expansion (CTE), thermoelastic deformations of the mirrors caused by power fluctuations of the laser, mechanical vibrations, pressure fluctuations or long-term material contraction (or creep). Temperature fluctuations and thermo-elastic mirror deformations effects can be easily mitigated at short time scales (f $>10 \mathrm{mHz}$, i.e. $<100 \mathrm{~s}$ ) by thermal shields (Dai et al., 2015; Sanjuan et al., 2015), low CTE materials and laser intensity stabilization, respectively. Mechanical vibrations in the spacecraft due to attitude actuators should not be relevant in the Hertz (second) range and, moreover, specific insensitive vibration mounts (Webster et al., 2007; Webster and Gill, 2011) can be used to further minimize these effects. Low pressure levels (and fluctuations) can be achieved by venting to space. Finally, creep effects at time scales of seconds to hundreds of seconds should not play any significant role. 
The noise sources related to the locking scheme are originated from shot-noise, RAM (Shen et al., 2015), electronic noise in the servo loops and actuators, parasitic etalons, etc. These are usually kept low when high-finesse cavities are used due to large signal-to-noise ratio (SNR) present in the discriminator. In addition, one of the important contributors, RAM-induced noise, can be actively corrected (Wong and Hall, 1985; Li et al., 2012; Zhang et al., 2014).

Optical cavities are expected to be an important part of future space missions involving laser interferometry: the GRACE-FO mission, which includes two cavities (one per spacecraft), should demonstrate frequency stability at the $10^{-15}[\mathrm{~s} / \mathrm{s}]$ level and pave the way for the next generation of gravity field missions based on laser interferometry. Based on the cubic cavity design (Webster and Gill, 2011), a setup on elegant breadboard level is currently integrated at DLR serving as a demonstrator for a future space-qualified cavity system. Beside its application within next generation Global Navigation Satellite Systems (GNSSs), cavity-based systems are also investigated with respect to next generation gravity missions, space-based tests of fundamental physics and the gravitational-wave detector LISA.

\subsection{Hybrid clock concept}

As mentioned above, Doppler-free saturation spectroscopy provides excellent long term frequency stability (for sample intervals larger than 100-1000 s) due to its low sensitivity to environmental conditions, especially with respect to temperature, while stabilization to optical cavities exhibits superior performance in the short time scales (milliseconds to few seconds). A potential scheme for combining both systems, resulting in a stable hybrid clock, is shown in Fig. 2 (left). It uses offset side-band locking (Thorpe et al., 2008) to keep a single laser head locked to two different reference systems at different time scales.
First, the laser is stabilized by using the Doppler-free spectroscopy unit. A pick-off of the laser output is sent to the cavity unity where an electro-optical modulator (EOM) generates the side-bands in order to bridge the gap between the iodine resonance frequency and the cavity fundamental modes. This corresponds to half of the cavity free spectral range, which for a $5 \mathrm{~cm}$ long cavity is as high as $1.5 \mathrm{GHz}$. On top of such modulation, the classic PDH locking scheme is implemented. The error signal from the PDH method is split into low- and-high frequency signals. The former is used to track the cavity fundamental mode, which shifts due to, e.g., temperature variations or creep, by adjusting the EOM frequency. The latter drives an AOM placed after the spectroscopy unit and tracks the cavity frequency stability in the short-time scales, i.e., in the thermalnoise limit regime.

The right side of Fig. 2 shows the results of a simulation with simplified subsystems in order to illustrate the benefits of a hybrid clock. The simulation assumes a $1064 \mathrm{~nm}$ solidstate Nd:YAG free running laser. Its frequency stability in Allan deviation is shown by the purple trace. The free running laser is locked to the iodine spectroscopy unit that provides the frequency stability shown in blue, which is the performance of the unit described in Section 2.1. The stability of the optical cavity corresponds to the red trace and it is assumed to have a frequency stability of $10^{-15}[\mathrm{~s} /$ s] for sample intervals smaller than $10 \mathrm{~s}$. The three systems (free running laser, spectroscopy unit and optical cavity) are simulated by generating time series of colored noise, which are fed into a time domain simulation of the hybrid clock scheme shown in the left panel of Figs. 1 and 2. The simulations do not consider sources of noise from the actuators (AOM and EOMs). The hybrid clock performance obtained from the simulations is given by the black trace, which exhibits the frequency stability of the spectroscopy unit for sample intervals larger than $50 \mathrm{~s}$ and the performance of the optical cavity at shorter sample intervals.
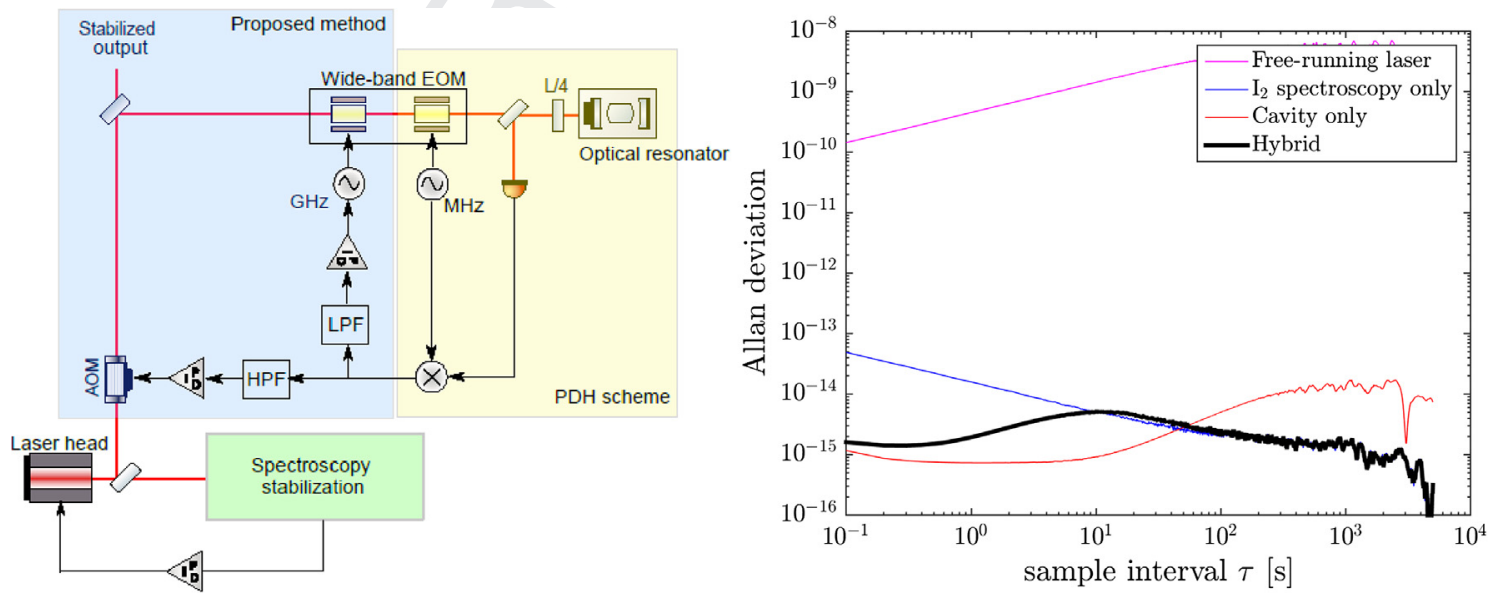

Fig. 2. Left: Hybrid clock scheme to combine a spectroscopy unit and an optical cavity. Right: Simulation results (with simplified systems) in square root of the Allan deviation. The black trace corresponds to the hybrid clock performance, which combines the long-term frequency stability of the iodine spectroscopy (red line) with the short-term frequency stability of a high finesse optical cavity (blue line). The purple trace represents the free-running laser. (For interpretation of the references to colour in this figure legend, the reader is referred to the web version of this article.) 
The hybrid clock concept is currently investigated at DLR and an experimental setup will be realized.

\section{Optical inter-satellite links}

Optical free-space links are an efficient and effective way to exchange point-to-point information characterized by low transmitted power, low weight, and high data rates. The main physical advantage of optical frequencies is the small transmitter beam divergence, which overcomes interference problems and radio frequency spectrum. However, the limited divergence introduces the challenge of keeping a precise alignment between the transmitter and the receiver.

The first optical transmission performed in space dates back to 1992, as part of the Galileo Optical EXperiment (GOPEX; Wilson et al., 1993), with the emission of optical pulses from Earth to a charge-coupled device (CCD) on the NASA Galileo spacecraft. The first optical communication between two space platforms took place in 2001, when an inter-satellite link was established between the optical terminals of the geostationary Advanced Relay and TEchnology MIssion Satellite (ARTEMIS) developed by Alenia for the European Space Agency (ESA) and the SPOT-4 LEO satellite developed by Matra Marconi Space for the National Centre for Space Studies (CNES - Centre National d'Études Spatiales). This experiment was performed within the Semiconductor Laser inter-satellite link EXperiment (SILEX; Fletcher et al., 1991), and demonstrated the feasibility of optical technologies in space for inter-satellite communications. The SILEX project, in which both laser terminals use on/off key modulation and direct detection of laser signals at $800 \mathrm{~nm}$, was the first European step in the direction of globally-distributed relay systems based on optical inter-satellite-links (TolkerNielsen and Oppenhauser, 2002). In 2005, inter-satellite links (ISLs) between the optical terminals onboard the Artemis satellite and the Japanese LEO satellite OICETS (Optical Inter-orbit Communications Engineering Test Satellite) were carried out, further demonstrating the potential of optical technologies in space (Takayama et al., 2007; Jono and Katsuyoshi, 2006).

An important advantage of optical links is the capability of relaying large data volumes between several space platforms. This can be exploited e.g. in missions that need to transmit a large amount of data to a single ground station: satellites that are not visible from the ground receiver may relay their data through another satellite capable of communicating with the ground station. Such a scheme is implemented in the European Data Relay System (EDRS): LEO satellites transmit data optically to a geostationary satellite, which sends it to the ground station in Ka-Band.

The first technology for this system was developed for LEO satellites, demonstrating inter-satellite links between TerraSAR-X of DLR and the Near-Field InfraRed Experiment (N-FIRE) of NASA in 2007 (Smutny et al., 2009). Both satellites carried a LCT developed by TESAT Spacecom. The link was based on Binary Phase Shift Keying
(BPSK) modulation and homodyne reception (Lange and Smutny, 2005). The TESAT LCT demonstrated data rates of $5.625 \mathrm{Gbit} / \mathrm{s}$, covering distances of up to $5500 \mathrm{~km}$. The LCT was further developed for geostationary satellites and integrated in the AlphaSat and EDRS satellites. This system became operational in November 2016, providing the SpaceDataHighway service (Airbus, 2018). NASA recently proposed an own data relay system, planned for launch in 2019: the Laser Communications Relay Demonstration (Israel et al., 2017). Private company SpaceX also proposed a constellation of thousands of LEO satellites connected by inter-satellite optical links.

A coherent optical ranging system is currently being tested on the GRACE-FO mission. In this experiment laser interferometry measurements of inter-satellite range rates in orbit will be demonstrated (Sheard et al., 2012).

Point-to-point optical inter-satellite coherent links are the only option for providing a channel to synchronize remote optical timing references at or below ps-level. In addition, the optical links provide a mean to perform sub-mm inter-satellite ranging. Both capabilities are further discussed in Section 4 in the framework of a GNSS system with inter-linked navigation satellites.

\subsection{Coherent communications}

General modulations, involving amplitude and phase modulation, require a coherent receiver with a local oscillator used to retrieve the code and carrier phases as well as the communication data. Coherent receivers can be categorized into homodyne, intradyne and heterodyne. In homodyne reception an optical phase-locked loop is required to receive the data, i.e. the phase and frequency of the local oscillator is tuned to match the received signal, with a relatively complex hardware. In fiber communications either intradyne or digital homodyne systems are usually employed, in which the phase-locked loop is implemented in the digital domain and the local oscillator is kept freerunning around the target frequency. In this case, the complexity is shifted to the processing unit. The last form of optical coherent reception is the heterodyne variant, in which the phase-locked loop is realized at radiofrequencies. In this case, the local oscillator is kept at a fixed frequency. This frequency, called intermediate frequency, is higher than the signal bandwidth. After the opto-electrical conversion, the data is retrieved at the intermediate frequency rather than in the base-band.

Homodyne coherent reception has the advantage of a high sensitivity. The first development on coherent reception for optical free-space communications was carried out by DLR and led to a world-record in sensitivity (Wandernoth, 1992). The system was based on BPSK and represented the first step towards the current technology in the LCTs developed by Tesat Spacecom. Since then, terminals for LEO and geostationary satellites have been developed, and numerous links between satellites have been successfully established (more than 20,000 as for early 
2019). Although a number of experiments also showed the feasibility of intradyne reception in free-space applications (Conroy et al., 2018; Geisler et al., 2017), homodyne reception is nowadays the only operational technology for intersatellite optical links. A homodyne receiver for the ISLs considered in this paper is discussed in detail in Section 4.2.

\subsection{Optical terminals for satellite communications}

Optical links are established via optical terminals on each end of the link. The terminals contain the optical assemblies that realize the pointing, acquisition and tracking of the laser beams. Furthermore, at the receiver, they inject the signal into an optical fiber. The key components for pointing, acquisition and tracking are the Coarse Pointing Assembly (CPA), which is used to steer the laser beams towards the remote receiver, and the Fine Pointing Assembly, which is typically implemented with a fast steering mirror, allowing for very precise and fast beam steering. Additionally, most satellite applications require the implementation of a point-ahead assembly to account for the relative dynamics of the platforms being linked: since the light speed is finite, the transmitter needs to account for the receiver motion in order to intercept its trajectory.

Other important components of the terminals are the optical subsystem, which handles the splitting between transmitted and received light; the optical amplifier, which is typically fiber-based and used to amplify the signal of a low-power seed laser; and the data receiver, which performs the opto-electrical conversion on the receiver side. Fig. 3 shows the Tesat LCT for geostationary applications with a $135 \mathrm{~mm}$ aperture. This LCT is used onboard Alphasat, as well as on EDRS satellites, and supports optical links between LEO and geostationary satellites. The LCT has been thoroughly tested and it is currently being used in an increasing number of applications, e.g. the EDRS is

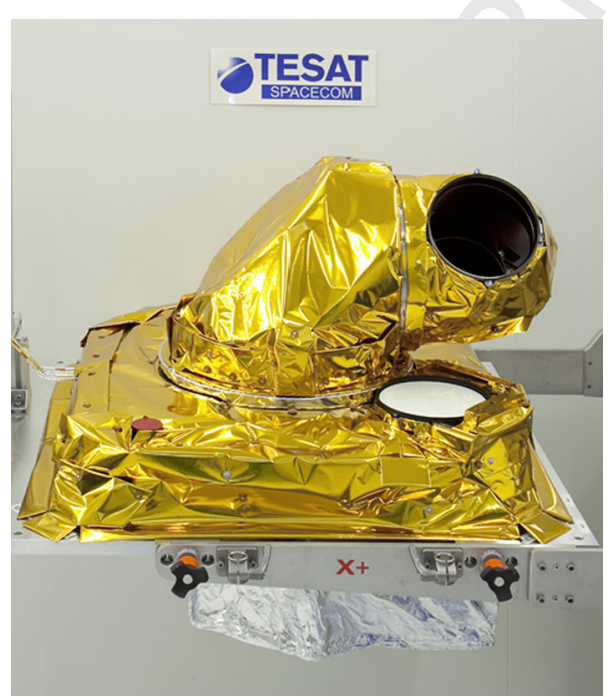

Fig. 3. Tesat Laser Communication Terminal for geostationary satellites (Picture: courtesy of Tesat-Spacecom GmbH and Co.). used within the European Union's Copernicus Earth observation program (Hauschildt et al., 2017). The technical requirements and construction of an optical terminal supporting the architecture presented in Section 4 are discussed in more details in Section 4.2.

\section{Exploitation of optical technologies in GNSS architectures and satellite geodesy}

\subsection{A novel architecture for GNSS}

Modern satellite navigation primarily relies on the broadcast of synchronized navigation signals from a constellation of MEO satellites. A navigation satellite system exploiting a network of optical frequency references synchronized via coherent inter-satellite laser links would enable establishing an ultra-stable time standard within the space segment in the optical domain. This stability can be transferred to radio frequencies by using frequency combs and employed to broadcast synchronized navigation signals to receivers on Earth via a classic navigation payload. This architecture is proposed in Günther (submitted for publication, 2018a, 2018b) and takes the name of Kepler. We give an overview of the main elements of this architecture.

A MEO segment, formed by a constellation of 24 MEO satellites (semi-major axis: 29,600 km), each equipped with cavity-stabilized lasers, is used to transmit legacy navigation signals towards the Earth as in any current GNSS. Each satellite is continuously connected to the two neighboring satellites in the same orbital plane via two-way inter-satellite laser links. A third nadir-pointing optical terminal on each MEO satellite enables non-continuous links to LEO satellites. The whole MEO segment is kept tightly synchronized via time transfer between optically linked satellites. The constellation is completed by a small LEO segment, in which six LEO satellites (semi-major axis: $7600 \mathrm{~km}$ ), each equipped with optical frequency references and three optical terminals, execute four main tasks. First, LEO satellites establish optical links to MEO satellites in different orbital planes in order to support the synchronization amongst all the constellation elements. In order to achieve $100 \%$ availability of a connection between three MEO planes, a single LEO satellite links to three MEO satellites all in different planes, or two LEO satellites link to two different pairs of MEO satellites on different planes. Two LEO satellites with three optical terminals each are sufficient if an unobstructed view of the whole upper hemisphere is available. The procedure to design the linking rule to coordinate links between the LEO and MEO segments and the design of low Earth orbits is given in more details in (Giorgi et al., in press). Second, LEO satellites are instrumental to the definition of a system time by carrying mid-term stable optical frequency references (e.g. Iodine references, see Section 2.1), which are employed to realize a mixed clock ensemble with target frequency stability at $10^{-15}[\mathrm{~s} / \mathrm{s}]$ for sample intervals between 0.1 and $106 \mathrm{~s}$ (more 
details are given in Section 4.3). Third, LEO satellites contribute to the precise determination of satellite orbits by providing atmosphere-free observations of the transmitted navigation signals via an upward-looking GNSS antenna and LEO-to-MEO inter-satellite laser ranging measurements (cf. Section 4.4). Lastly, LEO satellites provide an intra-system integrity layer by continuously monitoring the navigation signals and satellite biases without atmospheric disturbances. In order to continuously monitor the navigation signals with positive elevation angles, and add a certain degree of redundancy in support of system synchronization, six satellites are considered as baseline LEO constellation.

Fig. 4 shows the elements of the proposed space segment. Alternative space segments configurations may be devised by using geostationary satellites instead of LEO satellites for supporting inter-plane synchronization, or based on direct links between MEO satellites on different orbital planes. However, these options lack the broad spectrum of capabilities that a LEO segment would provide.

From a navigation perspective, reliable synchronization across the elements of the constellation in a quasi-inertial frame is an important requirement to obtain a precise navigation solution for users on Earth and its proximity. The tie with a terrestrial reference frame and time scale must be maintained in order to account for the Earth's rotation and to properly provide guidance to users navigating on
Earth. Differently from the approach in use in all currently active GNSSs, the Kepler infrastructure would only require a very limited set of ground stations to align with terrestrial space frames and time scales. From the standpoint of navigation performance, one ground station is in principle sufficient if a link between the space segment and the ground station can be continuously maintained over time, the coordinates of the station are fixed in the chosen terrestrial reference frame, and the Earth Rotation Parameters (ERPs) linking the terrestrial and the inertial frames are determined independently. In order to guarantee sufficient reliability and robustness of operations, a minimal set of regional ground station would be sufficient to operate the proposed system.

The limited size of the ground segment with respect to modern systems is made possible by the presence of LEO satellites, which aid orbit determination and provide signal monitoring capabilities. The ground station(s) also contributes to the definition of the system time, by hosting one or more active hydrogen masers that provide the long-term frequency stability necessary to align with the terrestrial Coordinated Universal Time (UTC) time scale.

The constellation-wide synchronization could be achieved by forming a clock ensemble with all optical reference on the satellites and microwave frequency references on ground (cf. Section 4.3). During normal operation, all the elements of the constellation share the measured offsets

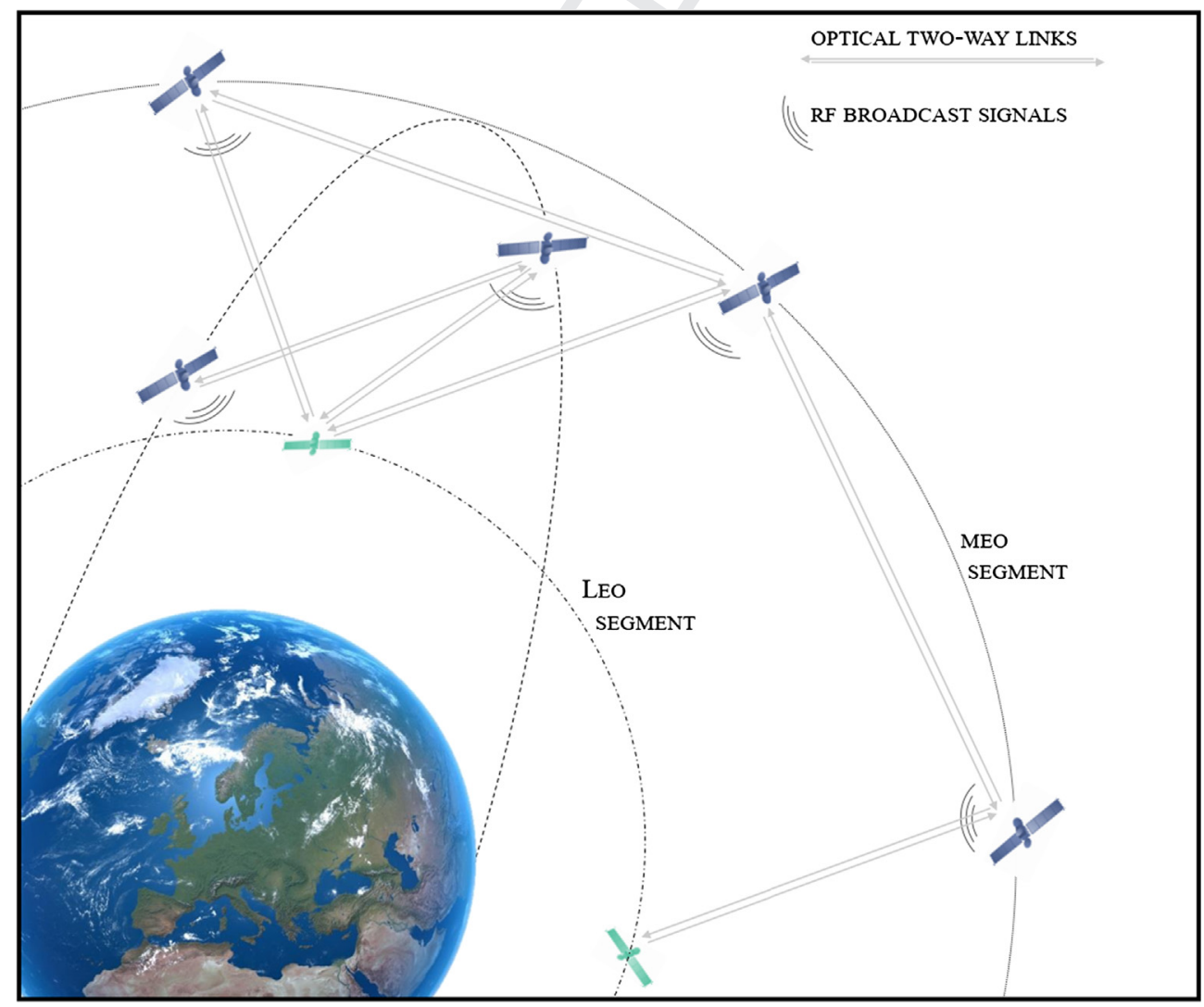

Fig. 4. Kepler constellation: MEO and LEO satellites carrying optical frequency references, interconnected by two-way laser links. In addition, a constellation of LEO satellites in lower orbits might exploit the synchronous broadcast navigation signals for high-accuracy geodetic applications. 
between linked frequency references, relayed with low latency via Optical Inter-Satellite Links (OISLs). The overall ensemble signal is then generated on each MEO satellite and it is used to time the navigation payload by steering the local oscillator towards the IEM: this is the technique used to realize the system-wide synchronous broadcast. The capabilities of the synchronization subsystem depend, among other aspects, on relativistic modeling and on the quality of real-time satellite orbit determination. Effects due to proper time and relativistic propagation at and between different spacetime locations in a non-spherical gravitational field need to be accounted for to a level below the required synchronization threshold: $10^{-16}[\mathrm{~s} / \mathrm{s}]$ fractional frequency stability is the foreseen target. This entails relativistic modeling to the order of $\mathrm{c}^{-3}$, inclusion of Earth geopotential harmonics beyond the $\mathrm{J}_{2}$ - moment, and additional celestial bodies influences (Blanchet et al., 2001; Kroese et al., 2018). The accurate modeling of relativistic effect for time and frequency transfer at ps-level sets the accuracy requirements on the orbit determination of linked satellites to $5-10 \mathrm{~cm}$ for positions and $10-15 \mu \mathrm{m} / \mathrm{s}$ for velocities (Giorgi et al., in press). The absolute distance between any two linked satellites needs to be known with higher precision, since $1 \mathrm{ps}$ synchronization precision requires spatial accuracy better than $0.3 \mathrm{~mm}$ : this is achieved via the optical ranging capability of the optical transceivers being developed (cf. Section 4.2).

Precise orbits are derived by processing inter-satellite ranging, measurements of broadcast navigation signals by LEO satellites and ground station(s), and potentially accelerations provided by interferometric inertial sensors (Guzmán et al., 2014). A first analysis of orbit determination in the Kepler system is provided in Michalak et al. (2018), showing an improvement of at least one order of magnitude with respect to current capabilities, due to the processing of navigation signals without atmospheric disturbances and the availability of accurate inter-satellite ranges. The precise orbits so computed serve as the basis for time and frequency transfer via two-way optical links between the constellation elements.

The system performance in terms of navigation is based on a combination of factors, and it is usually synthesized in the Signal-in-Space (user) Range Error (SISRE; Ch.1 in Teunissen and Montenbruck, 2017). This performance parameter measures the ranging accuracy provided by the navigation system without including error contributions from the user's specific receiver and environment (including atmospheric disturbances). The SISRE is mainly driven by the broadcast satellite orbit error and the satellite clocks error. In the Kepler system, the constellation-wide target synchronization is at $1 \mathrm{ps}$ level, which allows neglecting the satellite clock error, since this remains well below the L-band phase measurement error. It is shown in (Giorgi et al., in press) how the radial component of the satellite position could be estimated with accuracy at or below $1 \mathrm{~cm}$. This parameter is the key to improve satellite navigation and time dissemination - with $1 \mathrm{~cm}$ spatial accuracy converting into $33 \mathrm{ps}$ timing accuracy. In order to guarantee a SISRE at $1 \mathrm{~cm}$, the broadcast ephemeris must enable the user to compute the satellite orbits with the same level of accuracy. The navigation message in the Galileo Open Service can already accommodate an ephemeris update rate of $50 \mathrm{~s}$ in the F/NAV message and $30 \mathrm{~s}$ in the I/ NAV message (European Union, 2016). A Kepler-like architecture is suitable for taking full advantage of this rate, thanks to the possibility of relaying information to the whole constellation via the OISLs with low latency. Further ad-hoc investigations are undergoing to assess the extent of improvement in terms of SISRE that Kepler will bring to GNSS users. Table 1 provides a comparison between current GNSSs and the proposed architecture. The broadcast navigation signals are not discussed in detail, but it is worth pointing out that current signals already enable cm-level pseudorange tracking accuracy, e.g. Galileo's E5 altBOC (Tawk et al., 2012).

A constellation of inter-linked satellites can also form the basis of a wholly different approach to satellite navigation, enabling the realization of a relativistic navigation system, in which the satellite emission coordinates are used to form a space-based reference system (Gomboc et al., 2014). In such a system, the satellite orbits are determined through dissemination of each satellite proper time, and users estimate their own positions without the need to apply relativistic corrections, potentially improving and simplifying the computational steps involved in extracting position estimates (Cadez et al., 2010; Cadez et al., 2011; Gomboc et al., 2014).

\subsection{Optical transceivers and laser communication terminals for the Kepler architecture}

Each satellite in the system detailed in the previous section employs laser communication terminals coupled to optical transceivers. The latter are a common optical element to all satellites. Fig. 5 illustrates the top-level block diagram of an optical transceiver that realizes the coherent two-way optical links. A Pseudo Random Noise (PRN) sequence mixed with the data modulates the optical carrier and it is transmitted through the telescope. At the receiver side, a fiber-coupled balanced receiver is used to convert both ranging and communications signals to the electrical domain, by mixing the received signal with a local oscillator. Since the inter-satellite free-space path does not introduce any wave-front distortion, stable single-mode fiber coupling at the receiver is achieved. The frequency offset between a local oscillator locked to the incoming signal and the cavity-stabilized laser is continuously measured and accounted for in the subsequent processing. An optical phase-locked-loop drives the local oscillator, matching the frequency and phase of the received light. The phase delay of the local spread spectrum sequence is controlled by a delay-locked-loop (DLL) and determines the timing of the received signal code measurement. The SNR at the receiver is maximized by performing the correlation in 
Table 1

Outlook of current GNSSs space segments, ground segments and timing subsystems (Teunissen and Montenbruck, 2017; and references therein) compared to the proposed Kepler architecture. ${ }^{*}$ PHM: Passive Hydrogen Maser; ${ }^{* *}$ RAFS: Rubidium Atomic Frequency Standard.

\begin{tabular}{|c|c|c|c|c|c|}
\hline & GPS & Galileo & Glonass & BeiDou & Kepler \\
\hline $\begin{array}{l}\text { Nominal MEO } \\
\text { constellation } \\
\text { [additional } \\
\text { satellites] }\end{array}$ & 24 on 6 orbital planes & Walker $24 / 3 / 1$ & Walker 24/3/1 & $\begin{array}{l}\text { Walker } 27 / 3 / 1 \\
{[5 \mathrm{GEO}+3} \\
\text { IGSO }]\end{array}$ & $\begin{array}{l}\text { MEO: Walker } 24 / 3 / 1 \\
\text { LEO: Walker } 6 / 2 / 1\end{array}$ \\
\hline $\begin{array}{l}\text { Semi-major axis } \\
\text { (MEO) }\end{array}$ & $26,562 \mathrm{~km}$ & $29,600 \mathrm{~km}$ & $25,470 \mathrm{~km}$ & $27,870 \mathrm{~km}$ & $\begin{array}{l}\text { MEO: } 29,600 \mathrm{~km} \\
\text { LEO: } 7600 \mathrm{~km}\end{array}$ \\
\hline Eccentricity & $<0.02$ & $<0.003$ & $<0.01$ & 0 (nominal) & $<0.003$ \\
\hline Inclination & $55 \mathrm{deg}$ & $56 \mathrm{deg}$ & $65 \mathrm{deg}$ & $55 \mathrm{deg}$ & $\begin{array}{l}\text { MEO: } 56 \mathrm{deg} \\
\text { LEO: } 89.7 \mathrm{deg}\end{array}$ \\
\hline Orbital period & $11 \mathrm{~h} 58 \mathrm{~m}$ & $14 \mathrm{~h} 05 \mathrm{~m}$ & $11 \mathrm{~h} 14 \mathrm{~m}$ & $12 \mathrm{~h} 52 \mathrm{~m}$ & $\begin{array}{l}\text { MEO: } 14 \mathrm{~h} 5 \mathrm{~m} \\
\text { LEO: } 1 \mathrm{~h} 50 \mathrm{~m}\end{array}$ \\
\hline $\begin{array}{l}\text { Ground track } \\
\text { repeat cycle }\end{array}$ & $\begin{array}{l}1 \text { sidereal day } / 2 \\
\text { orbits }\end{array}$ & $\begin{array}{l}10 \text { sidereal days } / 17 \\
\text { orbits }\end{array}$ & $\begin{array}{l}\approx 8 \text { sidereal days/ } \\
17 \text { orbits }\end{array}$ & $\begin{array}{l}\approx 7 \text { sidereal days/ } \\
13 \text { orbits }\end{array}$ & $\begin{array}{l}\text { MEO: } 10 \text { sidereal days } / 17 \text { orbits } \\
\text { LEO: } 1 \text { sidereal day } / 13 \text { orbits }\end{array}$ \\
\hline $\begin{array}{l}\text { Available satellite } \\
\text { frequency } \\
\text { references } \\
\text { (frequency } \\
\text { stability, Allan } \\
\text { deviation) }\end{array}$ & $\begin{array}{l}\text { Cs }\left(10^{-11} @ 1 \mathrm{~s}, 10^{-13}\right. \\
\text { daily) } \\
\mathrm{Rb}\left(10^{-9} @ 1 \mathrm{~s}, 10^{-14}\right. \\
\text { daily })\end{array}$ & $\begin{array}{l}\operatorname{PHM}^{*}\left(10^{-11}-10^{-12}\right. \\
\text { @ } 1 \mathrm{~s}, \\
\left.7 \cdot 10^{-15} \text { daily }\right) \\
\text { RAFS }^{* *}\left(10^{-11}-10^{-12}\right. \\
\text { a } 1 \mathrm{~s} \text {, } \\
\left.3 \cdot 10^{-14} \text { daily }\right)\end{array}$ & $\begin{array}{l}\text { Cs }\left(10^{-11} @ 1 \mathrm{~s}, 10^{-}\right. \\
13 \text { daily }) \\
\mathrm{Rb}\left(10^{-9} @ 1 \mathrm{~s},\right. \\
\left.10^{-14} \text { daily }\right)\end{array}$ & PHM $^{*}, \mathrm{RAFS}^{* *}$ & $\begin{array}{l}\text { MEO: 'clock-less', } \\
\text { cavity-stabilized lasers } \\
\text { (below } 10^{-14} @ 1-10 \mathrm{~s} \text { ) } \\
\text { LEO: optical clocks } \\
\text { (target: below } 5 \cdot 10^{-15} @ \text { all sample } \\
\text { intervals) }\end{array}$ \\
\hline $\begin{array}{l}\text { Broadcast signals } \\
\text { (Open Service) }\end{array}$ & $\mathrm{L} 1, \mathrm{~L} 2, \mathrm{~L} 5$ & $\mathrm{E} 1, \mathrm{E} 5 \mathrm{a} / \mathrm{b}, \mathrm{E} 6$ & $\begin{array}{l}\text { L1, L2 (FDMA) } \\
\text { L1, L2, L5 } \\
\text { (CDMA) }\end{array}$ & $\mathrm{B} 1, \mathrm{~B} 2, \mathrm{~B} 3$ & $\begin{array}{l}\text { MEO: Legacy (modernized) L-band } \\
\text { signals } \\
\text { LEO: none }\end{array}$ \\
\hline
\end{tabular}

Center frequencies: L5, E5a (1176.45 MHz); E5b, B2 (1207.14 MHz); L2 (1227.60 MHz); B3 (1268.52 MHz); E6 (1278.75 MHz); B1 (1561.098 MHz); L1,E1 (1575.42 MHz)

\begin{tabular}{|c|c|c|c|c|c|}
\hline $\begin{array}{l}\text { Ground } \\
\text { monitoring } \\
\text { stations }\end{array}$ & $\begin{array}{l}1 \text { System Control } \\
\text { Centre (US) } \\
\text { Global network of } 16 \\
\text { monitoring sites }\end{array}$ & $\begin{array}{l}2 \text { Galileo Control } \\
\text { Centers (EU) } \\
\text { Global network } \\
\text { of }>20 \text { stations }\end{array}$ & $\begin{array}{l}1 \text { System Control } \\
\text { Centre (RU) } \\
18 \text { monitoring } \\
\text { stations all within } \\
\text { Russian territory }\end{array}$ & $\begin{array}{l}1 \text { Central Control } \\
\text { Station (PRC) } \\
32 \text { stations ( } 2 \\
\text { upload, } 30 \\
\text { monitoring) all } \\
\text { within PRC }\end{array}$ & 1 ground station plus redundancies \\
\hline $\begin{array}{l}\text { System time } \\
\text { realization }\end{array}$ & $\begin{array}{l}\text { Ensemble of a } \\
\text { network of ground } \\
\text { and satellite atomic } \\
\text { clocks }\end{array}$ & $\begin{array}{l}\text { Ground master } \\
\text { clock (AHM) at a } \\
\text { Galileo Control } \\
\text { Center }\end{array}$ & $\begin{array}{l}\text { Ensemble of } \\
\text { ground hydrogen } \\
\text { masers on ground }\end{array}$ & $\begin{array}{l}\text { Ensemble of }>0 \\
\text { ground AHMs }\end{array}$ & $\begin{array}{l}\text { Ensemble of } 24 \text { (MEO) }+6 \text { (LEO) } \\
\text { cavity-stabilized lasers, } 6 \text { Iodine clocks } \\
\text { (LEO) and (at least) one H-maser } \\
\text { (Ground) }\end{array}$ \\
\hline
\end{tabular}

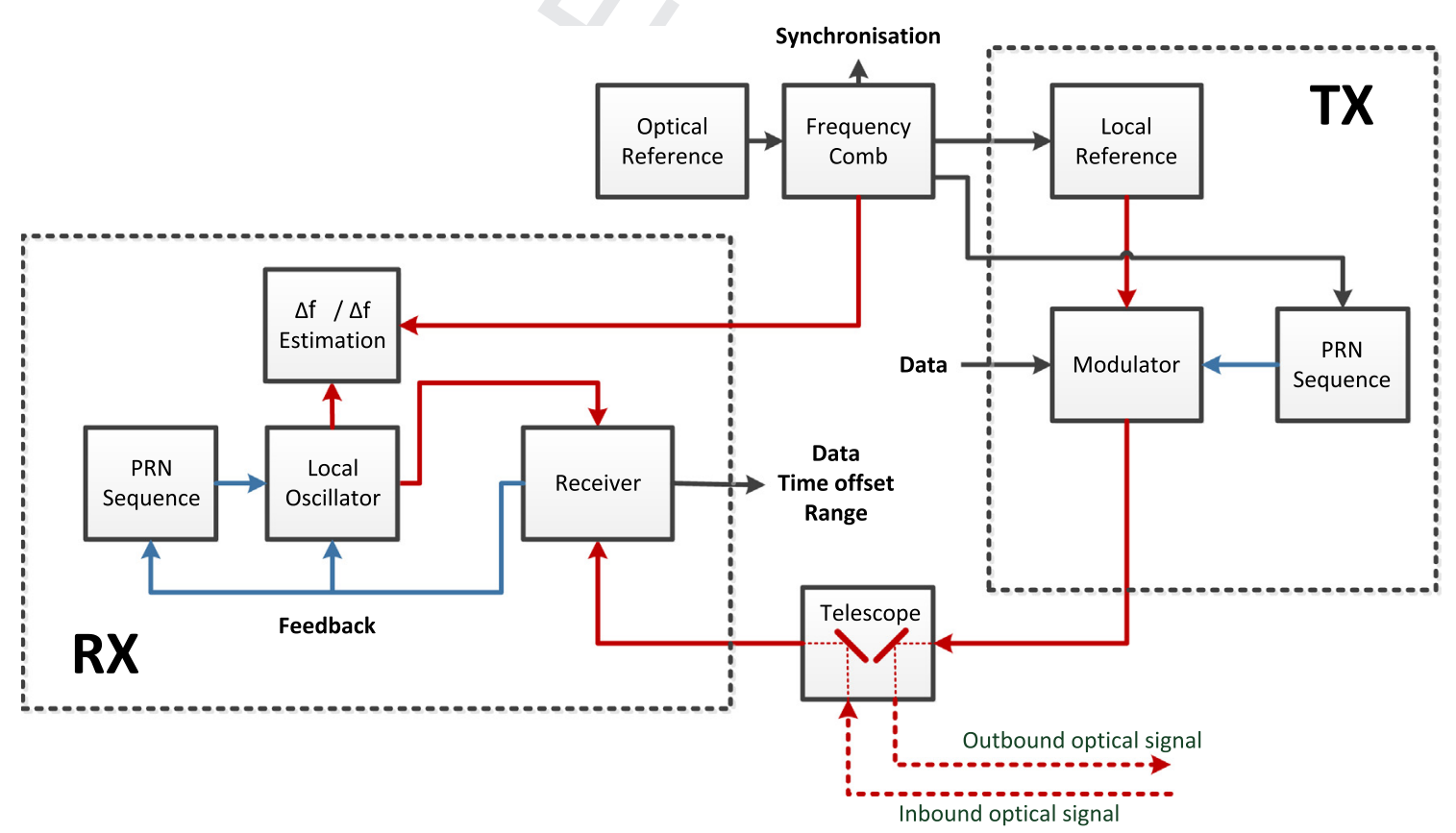

Fig. 5. Block diagram of a transceiver for optical ranging and communications. 
the optical domain. This scheme allows comparing the frequency and phase of the incoming signal to the reference cavity, and then measuring the time offset between neighboring satellites. The alignment of the received PRN code with the local replica enables ranging with accuracy in the order of $10^{2} \mu \mathrm{m}$ for a spreading sequence at 25.55 GigaChips per second. Finally, a BPSK modulation at 50 Megabit per second is used to transmit the data. A frequency comb is used to synchronize and/or compare the electrical and optical signals to the optical reference.

The second component of the laser communication system is the optical terminal. This is engineered on the basis of the relative dynamics between the platforms being linked. Three different optical terminal designs need then be addressed. A first CPA (type A) is employed to establish an optical link between neighboring MEO satellites. This unit needs not to cope with high relative dynamics and it must be capable of pointing either to the next satellite and to the second-next in case of malfunctions. A second CPA (type B) is used onboard MEO satellites to establish links to LEO satellites. This unit is characterized by a large field-of-view $\left(14.6^{\circ}\right.$ conical aperture from nadir). A third CPA (type C) is used onboard LEO satellites to establish links to MEO satellites, and it must be designed to cope with the relative high dynamics between MEO and LEO satellites (roughly $360^{\circ} / 90 \mathrm{~min}$ for tracking and 50 times faster for pointing). Fig. 6 shows the general terminal functional design layout of a MEO satellite, in which the aforementioned parts are explicitly illustrated.

The main impairments in an inter-satellite link are due to satellite attitude variations and platform vibrations, which need to be compensated by the fine pointing subsystem to avoid fluctuations of the received power. From the ranging accuracy point-of-view, platform vibrations introduce length jitter. Inertial sensors may be employed to minimize the impact of such vibrations. For the purpose of demonstrating the ISL concept, two optical terminals are being developed based on commercial off-the-shelf components (Poliak et al., 2018), and will be used to emulate the MEO to MEO coherent optical link (to scale) and to demonstrate ranging and communication capabilities. Fig. 7 shows one of the two optical transceivers being assembled at DLR.

\subsection{Mixed clock ensembles and system time}

The optical frequency references described in Section 2.1 exhibit superior short-term performance in terms of fractional frequency stability compared to their classical microwave counterparts, achieving stabilities down to $10^{-15}[\mathrm{~s} / \mathrm{s}]$ for $0.1-10 \mathrm{~s}$ sample intervals. In the Kepler architecture discussed in Section 4.1, these references are kept synchronized via the two-way optical links, and provide the basis for a synchronous broadcast of navigation signals. However, additional references are needed for aligning the system time to a terrestrial time scale of choice, e.g. UTC: while the cavity-stabilized lasers can provide high shortterm frequency stability, the provision of a stable system time scale requires complementing the system with oscillators characterized by high mid- and long-term frequency stability. Fig. 8 shows the measured performance of three different units given in terms of Overlapping Allan Deviation (OADEV): a cavity-stabilized laser (Optical Reference System - ORS -MenloSystems), an iodine-clock (cf. Section 2) and an Active Hydrogen Maser (AHM - IEM KVARZ, model CH1-75A) available at the DLR's Time Lab. Each clock type is characterized by different frequency stability behavior with peak performance located at different durations of the sample interval. It is then desirable to combine these different types of frequency references, profiting from the individual advantages of each unit. An ensemble of all frequency references in the system is a viable choice for generating the Kepler system time, as well as forming the basis for time and frequency synchronization amongst the elements of the constellation. In a clock ensemble, the phases of multiple frequency references are combined to produce a virtual ensemble signal, named Implicit Ensemble Mean (IEM), whose frequency stability surpasses each of its constituents (Brown, 1991). This signal can be generated by actively steering a numerically controlled oscillator, thus physically realizing the IEM of the ensemble. An example of time scale defined by an ensemble is the Global Positioning System Time, which is composed by a number of atomic clocks located within the ground GPS control segment and all the atomic frequency references onboard GPS satellites. One important feature of an ensemble is the capability of integrating real-time detec-

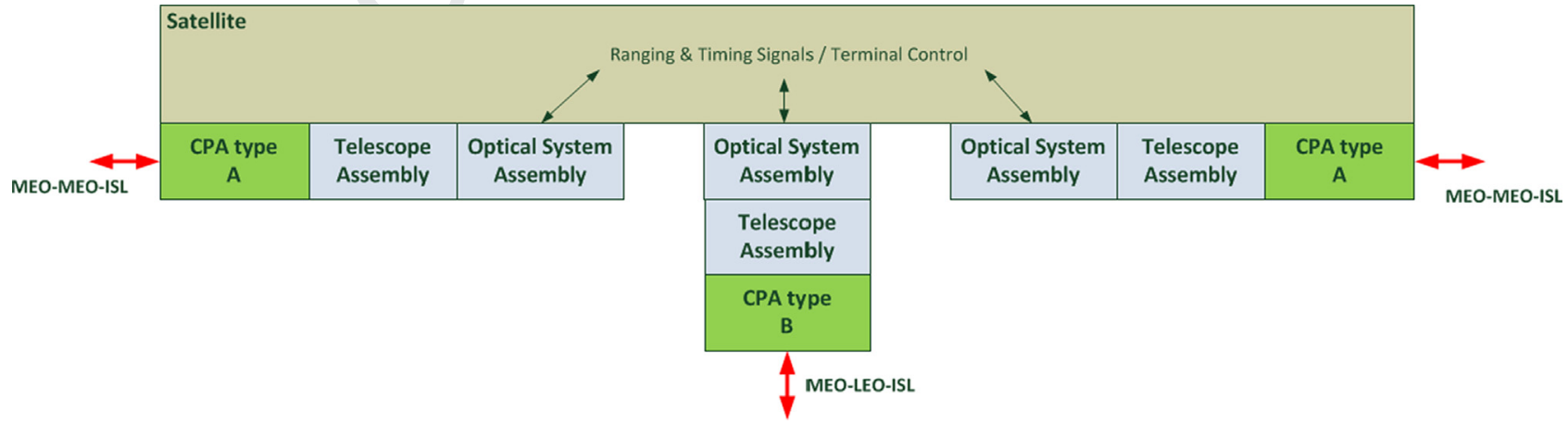

Fig. 6. Optical terminal configuration for MEO-satellites. 


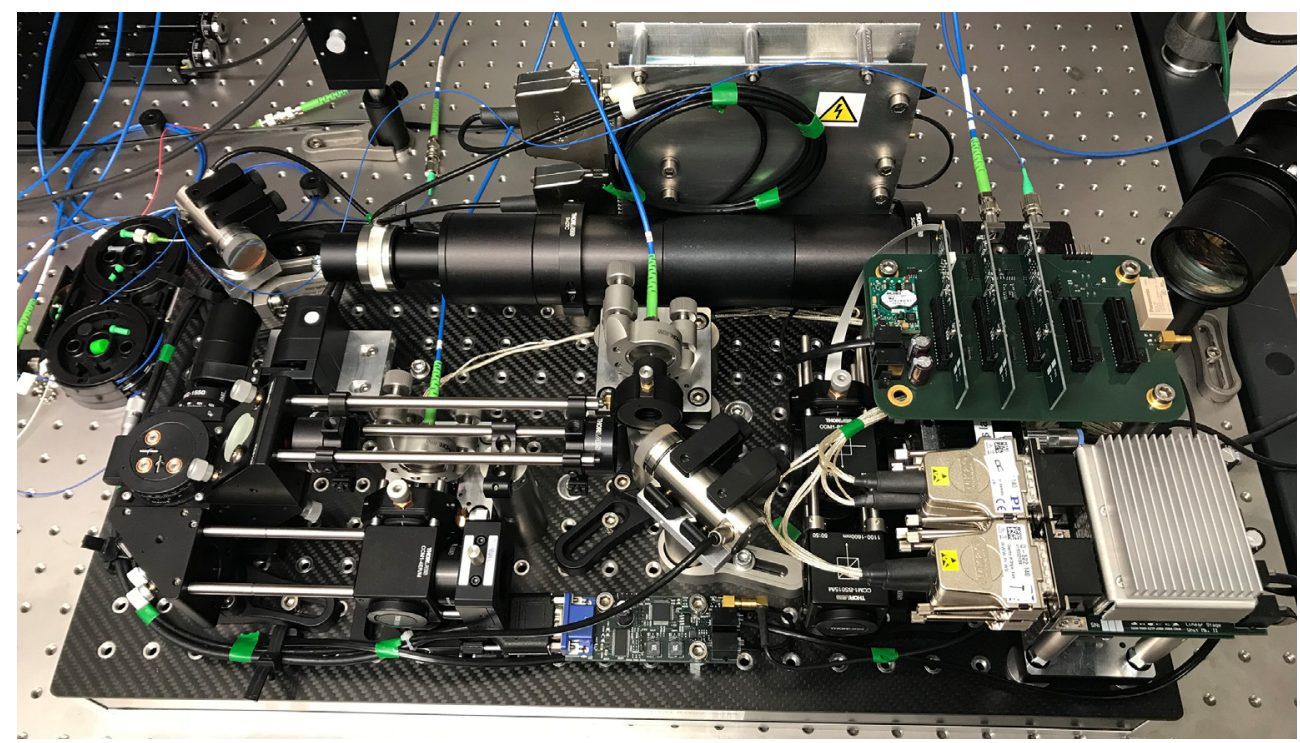

Fig. 7. The optical transceiver assembled at DLR.

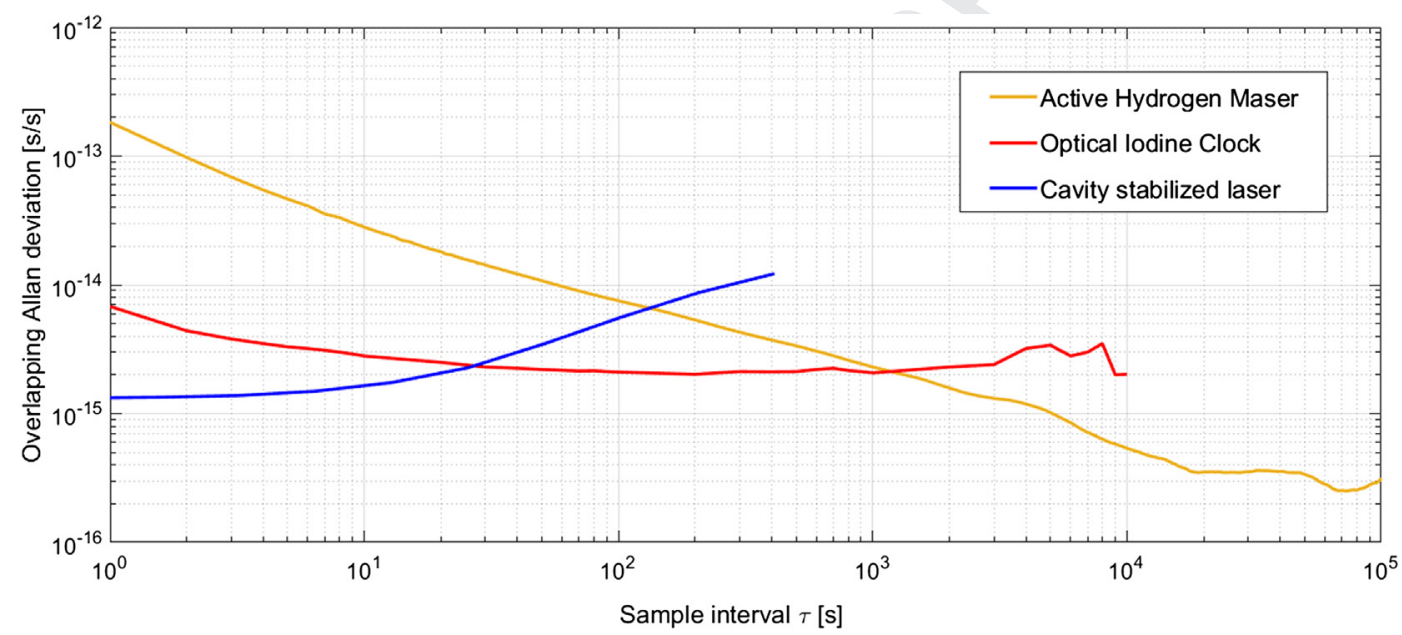

tion, isolation and adaptation techniques for protecting against failures of single clocks, thus enabling the provision of a robust time scale (Gödel and Furthner, 2017a).

The ensemble is computed by running a Kalman filter to predict future clock states based on clock models, which may be extended by Markov processes to take into account the pronounced flicker floor noise features of optical oscillators (Gödel et al., 2017b; Trainotti et al., 2019). These estimates are then used to weight the single clock contributions and to calculate an IEM, which exhibits better overall frequency stability than each of the individual clocks. Since a physical realization of the IEM thus generated is required for synchronizing the navigation messages broadcast by the satellites, a second Kalman filter is used to steer a timing reference towards the computed IEM (Schmidt et al., 2018a, 2019).
The system time in the Kepler system could then be defined by a mixed ensemble computed on all satellites, profiting from the improved robustness and frequency stability that characterizes heterogeneous ensembles. The mixed ensemble may consist of 30 cavity-stabilized lasers (24 on MEO satellites and 6 on LEO satellites), 6 Iodine clocks (one on each LEO satellite) and one Active Hydrogen Maser co-located with one ground station (Günther, submitted for publication, 2018a; Schmidt et al., 2018b). Fig. 9 shows an IEM generated by simulating the aforementioned ensemble of 37 frequency references. The measured OADEV (dashed lines) values are fitted to derive the parameters of two-state clock models that represent the expected behavior of the three clock types (solid lines). Based on these models, all the frequency references are simulated, and the IEM is computed. The IEM follows the 


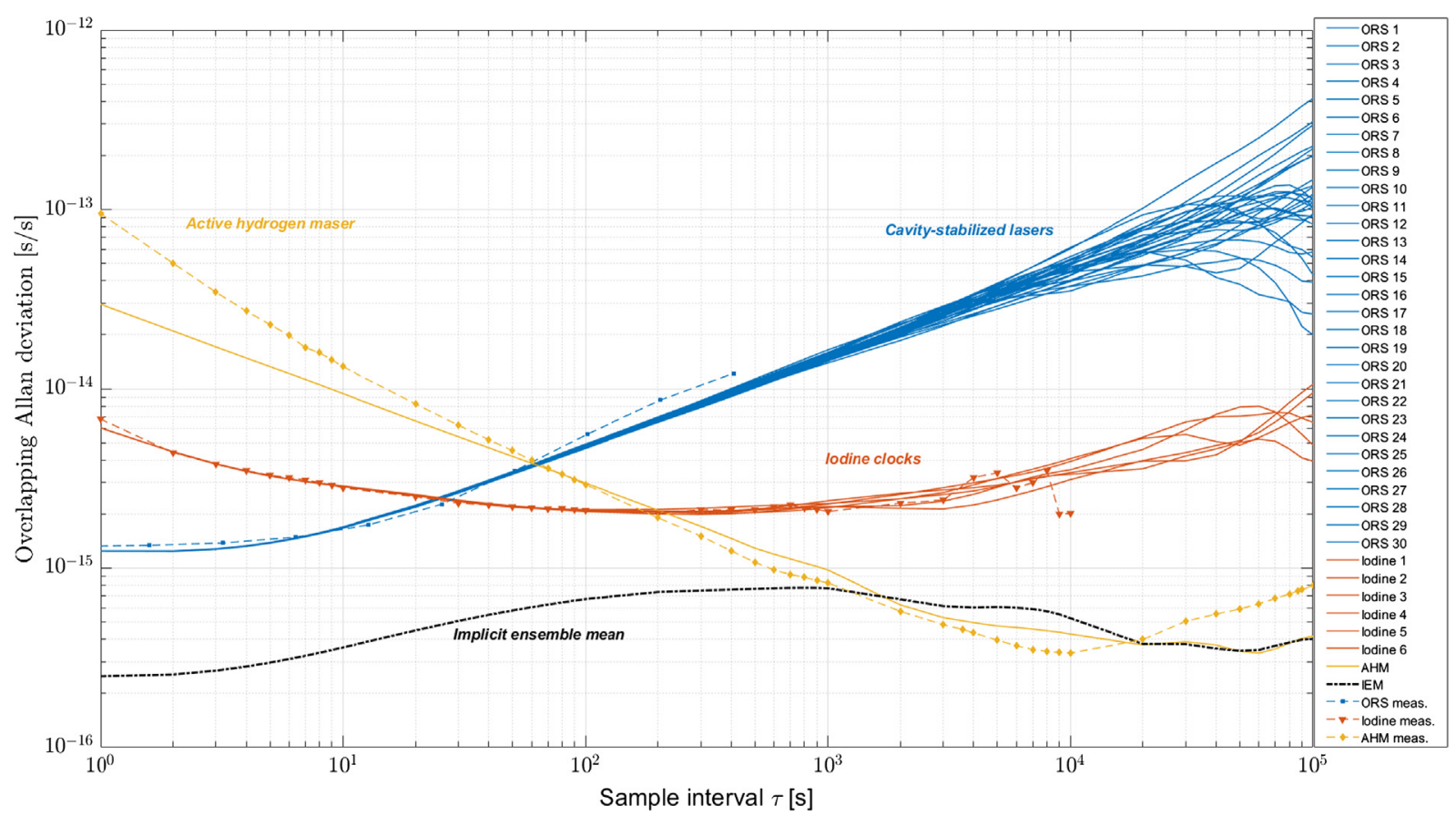

Fig. 9. Simulation of an Implicit Ensemble Mean (black line) computed using 30 cavity-stabilized lasers (continuous blue lines), six Iodine clocks (continuous red lines) and one active hydrogen Maser (continuous yellow line). Each simulated instance is based on a two-state clock model whose parameters are derived by fitting actual OADEV measurements (dashed lines). (For interpretation of the references to colour in this figure legend, the reader is referred to the web version of this article.)

best-performing clock of the ensemble for all sample intervals, showing frequency stability performance below the $10^{-15}[\mathrm{~s} / \mathrm{s}]$ level for the whole range of intervals analyzed. The robustness of the system is also significantly improved by using multiple units for each oscillator type. Since all oscillators are simultaneously contributing to the IEM, the latter is not severely degraded by isolated glitches or failures, such as phase or frequency jumps, or complete outages (Gödel and Furthner, 2017a).

The Kalman filter realizing the IEM is executed by each MEO satellite on the basis of differential measurements from all the distributed time offsets, relayed via OISLs (cf. Section 4.1) or via microwave carriers to/from the ground station (Günther, 2018a). By using the same propagation model, process noise and observation noise, all MEO satellites compute the same predicted IEM value at current epoch. The IEM is used to time the generation of the navigation signals from each satellite, and it constitutes the core of the real-time, constellation-wide synchronization scheme.

\subsection{System exploitation for geodesy and Earth observation}

The technologies and infrastructure discussed in previous sections enable key advancements in a variety of geodetic fields. In particular, we here briefly discuss potential impacts on the monitoring of the Earth gravity field, the provision of terrestrial reference frames, atmosphere sensing applications and GNSS reflectometry.
Several space-borne missions such as the NASA/GFZ missions GRACE/GRACE-FO (Tapley et al., 2004; Flechtner et al., 2014), the DLR missions TEXUS-51/53 and QUANTUS/MAIUS-1/JOKARIUS (Schkolnik et al., 2017; Döringshoff et al., 2019), and the Chinese mission Tiangong-2, have already demonstrated the feasibility of operating inter-satellite links, have successfully tested optical clocks components in a space environment and have illustrated their potential to benefit space geodesy. Next generation gravity field missions (NGGMs; Reubelt et al., 2014) dedicated to the retrieval of the Earth's gravity field will build up on the expertise acquired with the GRACE (2002-2017) and GRACE-FO (operative since May 2018) missions. Currently, different error sources (e.g. accelerometer data, background models, POD) limit the potential of precise inter-satellite ranging observations (Flechtner et al., 2016). Highly stable optical references, precise optical ranging with coherent OISLs and the large improvement in SISRE expected from GNSS architectures such as Kepler, carry the potential of largely improving the scope and reach of NGGMs, in particular with respect to spatial and temporal resolutions of the individual monthly models and the estimates of long term trends, enabling global monitoring of temporal gravity field variations and homogeneous accuracies at spatial scales below $300 \mathrm{~km}$, which is the current threshold for GRACE and GRACEFO. First simulation results have already shown that the orbit residuals of a GRACE-like LEO can be improved due to the envisioned SISRE in the Kepler system by a fac- 
tor of 7 -- from $27 \mathrm{~mm}$ to $4 \mathrm{~mm}$-- which in turn also significantly increases the capability to adjust low-degree gravity field coefficients, a known weakness of GRACE and GRACE-FO missions. The monthly gravity models of a GRACE-like single-pair mission would benefit in the complete spectrum by about 15\% (Murböck et al., 2019).

Future GNSS architectures capable of providing enhanced positioning and timing services will have an impact on the provision of global terrestrial reference frames (TRFs). These are the realization of a reference system defined by origin, orientation and scale, and are the metrological basis for almost all geodetic measurements associated with the Earth. Accurate and stable TRFs are highly relevant to reliably model and predict geophysical phenomena like the global sea level rise as one of the most significant examples with respect to climate change. The requirements of $1 \mathrm{~mm}$ accuracy and $1 \mathrm{~mm} /$ decade longterm stability set by the Global Geodetic Observing System (GGOS) are not yet fulfilled by the state-of-the-art TRFs. The latest official solution determined from a combination of GNSS, Doppler Orbitography and Radiopositioning Integrated by Satellite (DORIS), Satellite Laser Ranging (SLR), and Very Long Baseline Interferometry (VLBI) observations is the ITRF2014 (Altamimi et al., 2016). The accuracy of the origin of ITRF2014, realized by SLR, is $3 \mathrm{~mm}$ at reference epoch 2010.0 with respect to the previous realization ITRF2008. The scale of ITRF2014, realized from SLR and VLBI, shows a discrepancy of $1.37 \mathrm{ppb}$ (corresponding to $\sim 8.8 \mathrm{~mm}$ on the Earth's surface) at epoch 2010.0 between both techniques, which is still considered an unsolved problem. Thus far, GNSSs do not contribute to the realization of the origin and of the scale of the ITRF. Since the GGOS requirements are not met by state-of-the art TRFs, it is of high interest to investigate the potential improvement of the future GNSS constellations such as Kepler on the provision of TRF. First simulation results show that the standard deviations of the estimated geocenter as the realized origin of the TRF can be significantly improved by a factor of 14 , mainly due to the processing of navigation signals made available by the antennas on LEO satellites (cf. Section 4.1). The realization of mm-level ranging via the ISLs and synchronized clocks yields an improvement of smaller magnitude (Glaser et al., 2019). These results are obtained by simulating the whole Kepler constellation and processing its signals to determine the TRF, station coordinates, geocenter coordinates and Earth rotation parameters by imposing no-net-rotation and no-net-translation conditions on a globally distributed geodetic network of 124 sensing stations.

Observations of atmospheric parameters, and specifically of those characterizing the ionospheric and tropospheric layers, can be improved in different aspects. When navigation signals are used to 'sample' the ionosphere, the retrieval of ionospheric delays is limited by the accuracy of the satellite orbits and by group delays. Both aspects are greatly reduced in the Kepler system described in Section 4.1, where more precise satellite orbits are available and satellite-specific biases (group delays and antenna effects) can be constantly monitored from the LEO segment. Better ionospheric products have a direct impact on precise point positioning, navigation as well as on the performance of time and frequency transfer over continental scales (Hoque et al., 2017).

GNSS is also an established observation system for accurate water vapor monitoring (e.g., Bevis et al., 1992). Water vapor is the most volatile greenhouse gas, which partially drives weather systems and climate change; thus, weather forecasting, especially under severe weather conditions (e.g., such as heavy precipitation events and flash floods) is inherently linked to the ability to predict the spatial-temporal distribution of atmospheric water vapor. GNSS tropospheric estimates are an invaluable commodity for meteorological and climate studies (e.g., Alshawaf et al., 2018; Balidakis et al., 2018) as well as operational weather forecast (Gendt et al., 2004; Guerova et al., 2016; Zus et al., 2019a; Zus et al., 2019b). The frequency of severe weather events increases, as a repercussion of global warming (e.g., Kharin et al., 2018); hence the ability of GNSS observing systems should be adapted to cope with this increased need for accuracy. The transition from GPS-only to multi-GNSS data analysis provides improved tropospheric products (e.g., Li et al., 2015; Lu et al., 2016). In addition to increasing the ensemble of space vehicles used in GNSS analysis to infer water vapor distribution, the use of optical frequency references and inter-satellite links offers a distinct advantage. With an improvement of at least one order of magnitude in orbit determination of navigation satellites, and the uncertainty stemming from the frequency stability of satellite clocks being greatly reduced, the estimation of more accurate zenith delays and gradient vector components in the geodetic adjustment could be enhanced. For instance, the uncertainty of the zenith delays improves by $4-5 \%$ if highly synchronized clocks (at ps-level) are successfully realized; when compared to a scenario where a state-of-the-art single-system MEO-only constellation.

The opportunity to use a system such as Kepler for GNSS reflectometry could significantly advance ocean altimetry and foster an important field of geodesy. Reflectometry means, in this respect, that satellites of the system establish pairwise reflection links in bistatic radar configuration, see Martín-Neira et al. (2011). Earlier impact studies on GNSS reflectometry already indicated an improved detection of sub-mesoscale ocean phenomena such as oceanic eddies or tsunamis (Stosius et al., 2011). Altimetric measurements, based on the reflection links, will particularly benefit from reduced orbit uncertainties of the transmitting and receiving satellites (Semmling et al., 2016). Specifically, the LEO constellation in the Kepler system carries the potential to increase the spatial and temporal coverage of today's satellite altimeters if an additional set of nadir-pointing GNSS antennas is implemented. 


\section{Summary}

Optical frequency references and inter-satellite laser links will pave the way for a new generation of global satellite navigation systems, enhancing positioning and timing services on a global scale and improving a wide spectrum of satellite geodesy applications.

Optical oscillators capable of frequency stabilities below the $10^{-15}[\mathrm{~s} / \mathrm{s}]$ level for a wide range of sample intervals are being designed and tested in space. Combined with optical inter-satellite links capable of coherent two-way transmissions, already available and operative in space for communication purposes, the foundations of a space-based system of synchronized optical frequency references are being laid.

This manuscript provides an overview of the technical developments in both optical technologies, which can be exploited on a revised GNSS architecture to the purpose of achieving a high degree of synchronization between the elements of the satellite constellation. This novel architecture enables a more clear-cut separation between the space and time domains - which hinders current satellite navigation and geodesy applications - with a strong impact on orbit determination and time dissemination capabilities. If the MEO satellites are kept continuously synchronized, the navigation signals can be broadcast synchronously at or below picosecond-level. These aspects drive the signalin-space accuracy of the proposed Kepler system, potentially improving on the current GNSSs by at least one order of magnitude. An additional potential benefit of the proposed system is the capability of offering a fast and global integrity layer thanks to the continuous observations of atmosphere-free navigation signals from LEO satellites, which can relay any performance indicator with low latency. Also, LEO satellites carrying GNSS antennas offer a platform to calibrate the broadcast navigation signals in space.

Other than improving global satellite navigation and time dissemination, architectures such as Kepler carry the potential of improving orbit determination of communication and scientific satellites, Earth gravity field sensing, the provision of reference frames, and the remote sensing of Earth.

In the course of the coming months the construction of a technological demonstrator will be completed at the DLR premises, aiming at evaluating in a controlled environment the capabilities of optical ranging and communication devices in support of frequency- and time-transfer techniques between optical frequency references.

\section{Uncited reference}

Schuldt et al. (2016).

\section{Acknowledgments}

This work was carried out in the framework of project ADVANTAGE, funded in part by the "Impuls- und Ver- netzungsfond" of the Helmholtz Association (grant ZT0007, January 13, 2017). Assistance by G. Beyerle (GFZ) is gratefully acknowledged.

\section{References}

Acef, O., Clairon, A., Du Burck, F., Turazza, O., Djerroud, K., Holleville, D., et al., 2010. Nd:YAG Laser frequency stabilized for space applications. In: International Conference on Space Optics (ICSO), Rhodes, Greece.

Alshawaf, F., Zus, F., Balidakis, K., Deng, Z., Hoseini, M., Dick, G., Wickert, J., 2018. On the statistical significance of climatic trends estimated from GPS tropospheric time series. J. Geophys. Res.: Atmos. 123, 10967-10990.

Airbus, 2018. SpaceDataHighway. Retrieved June 2018, from https:// www.airbus.com/space/telecommunications-satellites/space-data-highway.html.

Altamimi, Z., Rebischung, P., Metivier, L., Collilieux, X., 2016. ITRF2014: a new release of the International Terrestrial Reference Frame modeling nonlinear station motions. J. Geophys. Res.: Solid Earth 121 (8), 6109-6131.

Argence, B., Halloin, H., Jeannin, O., Prat, P., Turazza, O., de Vismes, E., et al., 2010. Molecular laser stabilization at low frequencies for the LISA mission. Phys. Rev. D 81 (8), 082002.

Argence, B., Prevost, E., Lévèque, T., Le Goff, R., Bize, S., Lemonde, P., et al., 2012. Prototype of an ultra-stable optical cavity for space applications. Optics Exp. 20 (23), 25409-25420.

Arie, A., Byer, R., 1993. Laser heterodyne spectroscopy of 127 I2 hyperfine structure near 532 nm. J. Optical Soc. Am. B 10 (11), 1990-1997.

Armano, M. et al., 2016. Sub-femto-g free fall for space-based gravitational wave observatories: LISA pathfinder results. Phys. Rev. Lett. 116 (23), 231101.

Balidakis, K., Nilsson, T., Zus, F., Glaser, S., Heinkelmann, R., Deng, Z., Schuh, H., 2018. Estimating integrated water vapor trends from VLBI, GPS, and numerical weather models: sensitivity to tropospheric parameterization. J. Geophys. Res.: Atmos. 123, 6356-6372.

Bevis, M., Businger, S., Herring, T., Rocken, C., Anthes, R., Ware, R., 1992. GPS Meteorology: remote sensing of atmospheric water vapor using the Global Positioning System. J. Geophys. Res. 97 (D14), 15787-15801.

Bjorklund, G., 1980. Frequency-modulation spectroscopy: a new method for measuring weak absorptions and dispersions. Optics Lett. 5 (1), 1517.

Blanchet, L., Salomon, C., Teyssandier, P., Wolf, P., 2001. Relativistic theory for time and frequency transfer to order c-3. Astron. Astrophys. 370 (1), 320-329.

Bloom, B., Nicholson, T., Williams, J., Campbell, S., Bishof, M., Zhang, X., et al., 2014. An optical lattice clock with accuracy and stability at the 10-18 level. Nature 506, 71-75.

Brown, K., 1991. The theory of the GPS composite clock. In: Proceedings of the 4th International Technical Meeting of the Satellite Division of The Institute of Navigation (ION GPS), Albuquerque, NM, USA, pp. 223-242.

Cadez, A., Kostic, U., Delva, P., and Carloni, S., 2010. Mapping the Spacetime Metric with a Global Navigation Satellite System. ESA Final Report. European Space Agency.

Cadez, A., Kostic, U., Delva, P., Carloni, S., 2011. Mapping the Spacetime Metric with a Global Navigation Satellite System extension of study: Recovering of orbital constants using intersatellites links. ESA Final Report. European Space Agency.

Chen, Q.-F., Nevsky, A., Cardace, M., Schiller, S., Legero, T., Häfner, S., et al., 2014. A compact, robust, and transportable ultra-stable laser with a fractional frequency instability of 10-15. Rev. Sci. Instrum. 85 (11), 113107.

Cole, G., Zhang, W., Martin, M., Ye, J., Aspelmeyer, M., 2013. Tenfold reduction of Brownian noise in high-reflectivity optical coatings. Nat. Photon. 7, 644-650. 
Conroy, P., Surof, J., Poliak, J., Mata Calvo, R., 2018. Demonstration of 40 GBaud intradyne transmission through worst-case atmospheric turbulence conditions for geostationary satellite uplink. Appl. Opt. 57 (18), 5095-5101.

Dai, X., Jiang, Y., Hang, C., Bi, Z., Ma, L., 2015. Thermal analysis of optical reference cavities for low sensitivity to environmental temperature fluctuations. Optics Exp. 23 (4), 5134-5146.

Döringshoff, K., Schuldt, T., Kovalchuk, E., Stühler, J., Braxmaier, C., Peters, A., 2017. A flight-like absolute optical frequency reference based on iodine for laser systems at $1064 \mathrm{~nm}$. Appl. Phys. B 123 (6), 183.

Döringshoff, K., Gutsch, F., Schkolnik, V., Kürbis, C., Oswald, M., Pröbster, B., Kovalchuk, E., Bawamia, A., Smol, R., Schuldt, T., Lezius, M., Holzwarth, R., Wicht, A., Braxmaier, C., Krutzik, M., Peters, A., 2019. Iodine frequency reference on a sounding rocket. Phys. Rev. Appl. 11, 054068.

Drever, R., Hall, J., Kowalski, F., Hough, J., Ford, G., Munley, A., et al., 1983. Laser phase and frequency stabilization using an optical resonator. Appl. Phys. B 31 (2), 97-105.

European Union, 2016. Galileo - Open Service - Signal In Space Interface Control Document (OS SIS ICD V1.3). Accessible on https://www. gsc-europa.eu/electronic-library/programme-reference-documents.

Exertier, P., Samain, E., Martin, N., Courde, C., Laas-Bourez, M., Foussard, C., Guillemot, Ph., 2014. Time Transfer by Laser Link: data analysis and validation to the ps level. Adv. Space Res. 54 (11), 23712385.

Flechtner, F., Morton, P., Watkins, M., Webb, F., 2014. Status of the GRACE follow-on mission. In: Marti, U. (Ed.), Gravity, Geoid and Height Systems: Proceedings of the IAG Symposium GGHS2012, 9 12 October 2012, Venice, Italy, p. 117-121.

Flechtner, F., Neumayer, K., Dahle, C., Dobslaw, H., Fagiolini, E., Raimondo, J., et al., 2016. What can be expected from the GRACEFO Laser Ranging Interferometer for Earth science applications? Surveys Geophys. 37 (2), 453-470.

Fletcher, G., Hicks, T., Laurent, B., 1991. The SILEX optical interorbit link experiment. Electron. Commun. Eng. J., 273-279

Geisler, D., Yarnall, T., Schieler, C., Stevens, M., Robinson, B., Hamilton, S., 2017. Experimental demonstration of multi-aperture digital coherent combining over a $3.2-\mathrm{km}$ free-space link. In: Proceedings of the Free-Space Laser Communication and Atmospheric Propagation XXIX, (p. 10096OC). San Francisco, CA, USA.

Gendt, G., Dick, G., Reigber, C., Tomassini, M., Liu, M., Ramatschi, M., 2004. Near real time GPS water vapor monitoring for numerical weather prediction in Germany. J. Meteorol. Soc. Japan 82 (1B), 361370.

Giorgi, G., Kroese, B., Michalak, G., 2019. Future GNSS constellations with optical inter-satellite links. Preliminary space segment analyses. In: Proceedings of the IEEE Aerospace Conference. Big Sky, MT, USA (in press).

Glaser, S., Michalak, G., Koenig, R., Neumayer, K.H., Maennel, B., Schuh, H., 2019. Geocenter estimation with a future GNSS constellation. Geophysical Research Abstracts, 21(EGU2019-15486), EGU General Assembly 2019, Vienna, Austria.

Gödel, M., Furthner, J., 2017a. Robust ensemble time onboard a satellite. In: Proceedings of the 48th Annual Precise Time and Time Interval Systems and Applications Meeting (PTTI), Monterey, CA, USA, pp. $26-43$.

Gödel, M., Schmidt, T., Furthner, J., 2017b. Kalman filter approaches for a mixed clock ensemble. Joint Conference of the European Frequency and Time Forum and IEEE International Frequency Control Symposium (EFTF/IFCS). Besancon, France.

Gomboc, A., Horvat, M., and Kostic, U., 2014. Relativistic GNSS. ESAPECS final Report. European Space Agency.

Goujon, D., Rochat, P., Mosset, P., Boving, D., Perri, A., Rochat, J., et al., 2010. Development of the Space Active Hydrogen Maser for the ACES Mission. 24th European Frequency and Time Forum (EFTF2010), Noordwijk, Netherlands.
Guerova, G., Jones, J., Douša, J., Dick, G., Haan, S.d., Pottiaux, E., et al., 2016. Review of the state of the art and future prospects of the ground-based GNSS meteorology in Europe. Atmos. Measur. Tech. 9 (11), 5385-5406.

Günther, C., 2018. Kepler - a concept for a future system for navigation, geodesy and metrology. J. Navigation (submitted for publication).

Günther, C., 2018a. Kepler - Satellite Navigation without Clocks and Infrastructure. In: Proceedings of the ION GNSS + 2018, Miami, FL, USA, pp. 849-856.

Kepler - satellite navigation system description and validation. In: Proceedings of the 9th Workshop on Satellite Navigation Technologies (NAVITEC 2018), European Space Research and Technology Centre (ESTEC), Noordwijk, The Netherlands.

Guzmán, F., Kumanchik, L., Pratt, J., Taylor, J., 2014. High sensitivity optomechanical reference accelerometer over $10 \mathrm{kHz}$. Appl. Phys. Lett. 104 (22) 221111.

Häfner, S., Falke, S., Grebing, C., Vogt, S., Legero, T., Merimaa, M., et al., 2015. 8x10-17 fractional laser frequency instability with a long room-temperature cavity. Optics Lett. 40 (9), 2112-2115.

Hauschildt, H., Mezzasoma, S., Moeller, H., Witting, M., Herrmann, J., 2017. European data relay system goes global. In: Proceedings of the 2017 IEEE International Conference on Space Optical Systems and Applications (ICSOS), pp. 15-18.

Hong, F.-L., Ishikawa, J., Bi, Z.-Y., Zhang, J., Seta, K., Onae, A., et al., 2001. Portable I2-stabilized Nd:YAG laser for international comparisons. IEEE Trans. Instrum. Measur. 50 (2), 486-489.

Hoque, M., Jakowski, N., Berdermaan, J., 2017. Transionospheric Microwave Propagation: Higher-Order Effects up to $100 \mathrm{GHz}$. In: Costanzo, S. (Ed.), Wave Propagation Concepts for Near-Future Telecommunication Systems. IntechOpen.

Israel, D., Edwards, B., Staren, J., 2017. Laser Communications Relay Demonstration (LCRD) update and the path towards optical relay operations. Proceedings of the IEEE Aerospace Conference. Big Sky, MT, USA.

Jiang, Y., Ludlow, A., Lemke, N., Fox, R., Sherman, J., Ma, L.-S., et al., 2011. Making optical atomic clocks more stable with 10-16-level laser stabilization. Nat. Photon. 5, 158-161.

Jin, L., Jiang, Y., Yao, Y., Yu, H., Bi, Z., Ma, L., 2018. Laser frequency instability of $2 \mathrm{e}-16$ by stabilizing to 30 -cm-long Fabry-Perot cavities at 578 nm. Optics Exp. 26 (14), 18699.

Jono, T., Katsuyoshi, A., 2006. The OICETS Missions. In: Proceedings of the Sixth International Conference on Space Optics (ESA/CNES ICSO), (p. id.86). ESTEC, Nordwijk, The Netherlands.

Kessler, T., Hagemann, C., Grebing, C., Legero, T., Sterr, U., Riehle, F., et al., 2012. A sub-40-mHz-linewidth laser based on a silicon singlecrystal optical cavity. Nat. Photon. 6, 687-692.

Kharin, V.V., Flato, G.M., Zhang, X., Gillett, N.P., Zwiers, F., Anderson, K.J., 2018. Risks from climate extremes change differently from $1.5^{\circ} \mathrm{C}$ to $2.0^{\circ} \mathrm{C}$ depending on rarity. Earth's Future 6, 704-715.

Kroese, B., Giorgi, G., Günther, C., 2018. Relativistic corrections for intersatellite frequency transfer. Proceedings of the 32nd European Frequency and Time Forum (EFTF), Torino, Italy.

Lange, R., Smutny, B., 2005. Optical inter-satellite links based on homodyne BPSK modulation: heritage, status, and outlook. Proceedings of the Free-Space Laser Communication Technologies XVII, San Jose, CA, USA.

Leibrandt, D., Thorpe, M., Bergquist, J., Rosenband, T., 2011a. Field-test of a robust, portable, frequency-stable laser. Optics Exp. 19 (11), 10278-10286.

Leibrandt, D., Thorpe, M., Notcutt, M., Drullinger, R., Rosenband, T., Bergquist, J., 2011b. Spherical reference cavities for frequency stabilization of lasers in non-laboratory environments. Optics Exp. 19 (4), 3471-3482.

Lezius, M., Wilken, T., Deutsch, C., Giunta, M., Mandel, O., Thaller, A., Schkolnik, V., Schiemangk, M., Dinkelaker, A., Kohfeldt, A., Wicht, A., Krutzik, M., Peters, A., Hellmig, O., Duncker, H., Sengstock, K., Windpassinger, P., Lampmann, K., Hülsing, T., Hänsch, T.W., 
Holzwarth, . Space-borne frequency comb metrology. Optica 3 (12), 1381-1387.

Li, L., Liu, F., Wang, C., Chen, L., 2012. Measurement and control of residual amplitude modulation in optical phase modulation. Rev. Sci. Instrum. 83, 043111.

Li, X., Zus, F., Lu, C., Dick, G., Ning, T., Ge, M., et al., 2015. Retrieving of atmospheric parameters from multi-GNSS in real time: validation with water vapor radiometer and numerical weather model. J. Geophys. Res.: Atmos. 120 (14), 7189-7204.

Lu, C., Li, X., Ge, M., Heinkelmann, R., Nilsson, T., Soja, B., et al., 2016. Estimation and evaluation of real-time precipitable water vapor from GLONASS and GPS. GPS Solut. 20 (4), 703-713.

Ludlow, A., Boyd, M., Ye, J., Peik, E., Schmidt, P., 2015. Optical atomic clocks. Rev. Modern Phys. 87 (2), 637-701.

Ludlow, A., Huang, X., Notcutt, M., Zanon-Willette, T., Foreman, S., Boyd, M., et al., 2007. Compact, thermal-noise-limited optical cavity for diode laser stabilization at 1x10-15. Optics Lett. 32 (6), 641-643.

Martín-Neira, M., D’Addio, S., Buck, C., Floury, N., Prieto-Cerdeira, R., 2011. The PARIS ocean altimeter in-orbit demonstrator. IEEE Trans. Geosci. Remote Sensing 49 (6), 2209-2237.

Matei, D., Legero, T., Grebing, C., Häfner, S., Lisdat, C., Weyrich, R., et al., 2016. A second generation of low thermal noise cryogenic silicon resonators. J. Phys.: Conf. Series 723, 012031

Matei, D., Legero, T., Häfner, S., Grebing, C., Weyrich, R., Zhang, W., et al., 2017. $1.5 \mu \mathrm{m}$ Lasers with Sub-10 mHz Linewidth. Phys. Rev. Lett. 118 (26), 26302.

Meng, W., Zhang, H., Huang, P., Wang, J., Zhang, Z., Liao, Y., Ye, Y., Hu, W., Wang, Y., Chen, W., Yang, F., Prochazka, I., 2012. Design and experiment of onboard laser time transfer in Chinese Beidou navigation satellites. Adv. Space Res. 51 (6), 951-958.

Michalak, G., Neumayer, K.H., Koenig, R., 2018. Precise orbit determination with inter-satellite links and ultra-stable time for a future satellite navigation system. In: Proceedings of the ION GNSS+ 2018, Miami, FL, USA, pp. 968-1001.

Musha, M., Kanaya, T., Nakagawa, K., Ueda, K., 2000. The short- and long-term frequency stabilization of an injection-locked $\mathrm{Nd}$ :YAG laser in reference to a Fabry-Perot cavity and iodine saturated absorption line. Optics Commun. 183 (1-4), 165-173.

Murböck, M., Dahle, C., Michalak, G., Neumayer, K., König, K., Flechtner, F., 2019. Impact of inter-satellite links and ultra-stable clocks within future GNSS constellations on gravity field parameters. Geophysical Research Abstracts, vol. 21, EGU2019-16136, 2019, EGU General Assembly 2019.

Nicholson, T., Martin, M., Williams, J., Bloom, B., Bishof, M., Swallows, M., et al., 2012. Comparison of two independent Sr optical clocks with $1 \times 10^{-17}$ stability at $10^{3} \mathrm{~s}$. Phys. Rev. Lett. 109 (23), 230801.

Notcutt, M., Ma, L.-S., Ye, J., Hall, J., 2005. Simple and compact 1-Hz laser system via an improved mounting configuration of a reference cavity. Optics Lett. 30 (14), 1815-1817.

Nyholm, K., Merimaa, M., Ahola, T., Lassila, A., 2003. Frequency stabilization of a diode-pumped Nd:YAG laser at $532 \mathrm{~nm}$ to iodine by using third-harmonic technique. IEEE Trans. Instrum. Measur. 52 (2), $284-287$.

Poliak, J., Mata Calvo, R., Surof, J., 2018. Laboratory demonstrator of optical inter-satellite links for the Kepler system. In: Proceedings of the ION GNSS+ 2018, Miami, FL, USA, pp. 861-867.

Ressel, S., Gohlke, M., Rauen, D., Schuldt, T., Kronast, W., Mescheder, U., et al., 2010. Ultra-stable assembly and integration technology for ground- and space-based optical systems. Appl. Optics 49 (22), 4296 4303.

Reubelt, T., Sneeuw, N., Iran Pour, S., Hirth, M., Fichter, W., Müller, J., 2014. Future Gravity Satellite Missions. Advanced Technologies in Earth Sciences, GEOTECHNOLOGIEN Science Report No. 20. Springer-Verlag, Berlin Heidelberg.

Sanjuan, J., Gürlebeck, N., Braxmaier, C., 2015. Mathematical model of thermal shields for long-term stability optical resonators. Optics Exp. 23 (14), 17892-17908.
Schkolnik, V., Döringshoff, K., Gutsch, F., Oswald, M., Schuldt, T., Braxmaier, C., et al., 2017. JOKARUS - design of a compact optical iodine frequency reference for a sounding rocket mission. EPJ Quantum Technol. 4 (1), 9.

Schmidt, C., Fuchs, C., 2017. The OSIRIS program - first results and outlook. In: Proceedings of the IEEE International Conference on Space Optical Systems and Applications (ICSOS), Naha, Japan, pp. $19-22$.

Schmidt, T., Gödel, M., Furthner, J., 2018a. Investigation of pole placement technique for clock steering. In: Proceedings of the ION Precise Time and Time Interval Meeting, Reston, VA, USA, pp. 22-29.

Schmidt, T., Trainotti, C., Isoard, J., Giorgi, G., Furthner, J., Günther, C., 2018b. Composite clock algorithms for system time in global navigation satellite systems. In: Proceedings of the ION GNSS+ 2018, Miami, FL, USA, pp. 963-967.

Schmidt, T.D., Trainotti, C., Furthner, J., 2019. Comparing clock steering technique performances in simulations and measurements. In: Proceedings of the 50th Annual Precise Time and Time Interval Systems and Applications Meeting, Reston, VA, USA, pp. 265-283.

Schreiber, K.U., Prochazka, I., Lauber, P., Hugentobler, U., Schäfer, W., Cacciapuoti, L., Nasca, R., 2010. Ground-based demonstration of the European Laser Timing (ELT) experiment. IEEE Trans. Ultrason., Ferroelect., Frequency Control 57 (3), 728-737.

Schuldt, T., Döringshoff, K., Milke, A., Sanjuan, J., Gohlke, M., Kovalchuk, E., et al., 2016. High-performance optical frequency references for space. J. Phys.: Conf. Series 723, 012047.

Schuldt, T., Döringshoff, K., Kovalchuk, E., Keetman, A., Pahl, J., Peters, A., et al., 2017. Development of a compact optical absolute frequency reference for space with 10-15 instability. Appl. Optics 56 (4), 11011106.

Schuldt, T., Oswald, M., Gohlke, M., Sanjuan, J., Abich, K., Döringshoff, K., Kovalchuk, E., Peters, A., Braxmaier, C., 2018. Optical Clock Technologies for Future GNSS. In: Proceedings of the ION GNSS+ 2018, Miami, FL, USA, pp. 857-860.

Semmling, A.M., Leister, V., Saynisch, J., Zus, F., Heise, S., Wickert, J., 2016. A phase-altimetric simulator: studying the sensitivity of Earthreflected GNSS signals to ocean topography. IEEE Trans. Geosci. Remote Sensing 54 (11), 6791-6802.

Sheard, B.S., Heinzel, G., Danzmann, K., Shaddock, D.A., Klipstein, W. M., Folkner, W.M., 2012. Inter-satellite laser ranging instrument for the GRACE follow-on mission. J. Geodesy 86 (12), 1083-1095.

Shen, H., Li, L., Bi, J., Wang, J., Chen, L., 2015. Systematic and quantitative analysis of residual amplitude modulation in PoundDrever-Hall frequency stabilization. Phys. Rev. A 92 (6), 063809.

Shirley, J., 1982. Modulation transfer process in optical heterodyne saturation spectroscopy. Optics Lett. 7 (11), 537-539.

Smutny, B., Fields, R., Lunde, C., Skoog, J., Muhlnikel, G., Sterr, U., et al., 2009. NFIRE and TerraSAR-X laser communication tests: ground- breaking results for inter-satellite and space-to-ground links. Proceedings of the 27th IET and AIAA International Communications Satellite Systems Conference (ICSSC), Edinburgh, UK.

Stosius, R., Beyerle, G., Hoechner, A., Wickert, J., Lauterjung, J., 2011. The impact on tsunami detection from space using GNSS-reflectometry when combining GPS with GLONASS and Galileo. Adv. Space Res. 47 (5), 843-853.

Surof, D., Poliak, J., Mata-Calvo, R., 2017. Demonstration of intradyne BPSK optical free-space transmission in representative atmospheric turbulence conditions for geostationary uplink channel. Optics Lett. 42 (11), 2173-2176.

Swierad, D., Häfner, S., Vogt, S., Venon, B., Holleville, D., Bize, S., et al., 2016. Ultra-stable clock laser system development towards space applications. Sci. Rep. 6, 33973.

Takayama, Y., Jono, T., Koyama, Y., Kura, N., Shiratama, K., Demelenne, B., Sodnik, Z., Bird, A., Arai, K., 2007. Observation of atmospheric influence on OICETS inter-orbit laser communication demonstrations Free-Space Laser Communications VII. Majumdar, A. K. \& Davis, C. C. (Eds.) SPIE, 2007, 6709, 67091B. 
Tapley, B.D., Bettadpur, S., Watkins, M., Reigber, C., 2004. The gravity recovery and climate experiment: mission overview and early results. Geophys. Res. Lett. 31, L9607.

Tawk, Y., Botteron, C., Jovanovic, A., Farine, P.-A., 2012. Analysis of Galileo E5 and E5ab code tracking. GPS Solut. 16 (2), 243-258.

Teunissen, P., Montenbruck, O., 2017. Springer Handbook of Global Navigation Satellite Systems. Springer International Publishing.

Thompson, R., Folkner, W., de Vine, G., Klipstein, W., McKenzie, K., Spero, R., et al., 2011. A flight-like optical reference cavity for GRACE follow-on laser frequency stabilization. In: Joint Conference of the IEEE International Frequency Control and the European Frequency and Time Forum (FCS) Proceedings, San Francisco, CA, USA, pp. 1-3.

Thorpe, J., Numata, K., Livas, J., 2008. Laser frequency stabilization and control through offset sideband locking to optical cavities. Optics Exp. 16 (20), 15980-15990.

Tolker-Nielsen, T., Oppenhauser, G., 2002. In-orbit test result of an operational optical intersatellite link between ARTEMIS and SPOT4, SILEX. In: Proceedings of SPIE 4635, Free-Space Laser Communication Technologies XIV.

Trainotti, C., Schmidt, T.D., Furthner, J., 2019. Comparison of clock models in view of clock composition, clock steering and measurement fitting. In: Proceedings of the 50th Annual Precise Time and Time Interval Systems and Applications Meeting, Reston, VA, USA, pp. 265-283.

Ushijima, I., Takamoto, M., Das, M., Ohkubo, T., Katori, H., 2015. Cryogenic optical lattice clocks. Nat. Photon. 9, 185-189.

Vogt, S., Lisdat, C., Legero, T., Sterr, U., Ernsting, I., Nevsky, A., et al., 2011. Demonstration of a transportable $1 \mathrm{~Hz}$ linewidth laser. Appl. Phys. B 104 (4), 741.
Wandernoth, B., 1992. 20 photon/bit 565 Mbit/s PSK homodyne receiver using synchronisation bits. Electron. Lett. 28 (4), 387-388.

Webster, S., Gill, P., 2011. Force-insensitive optical cavity. Optics Lett. 36 (18), 3572-3574.

Webster, S., Oxborrow, M., Gill, P., 2007. Vibration insensitive optical cavity. Phys. Rev. A 75, 011801.

Webster, S., Pugla, S., Millo, J., Oxborrow, M., Gill, P., 2008. Thermalnoise-limited optical cavity. Precision Electromagnetic Measurements Digest (CPEM 2008). Broomfield, CO, USA.

Wilson, K., Lesh, J., Yan, T.-Y., 1993. GOPEX: a laser uplink to the Galileo spacecraft on its way to Jupiter. In: Proceedings of Optics, Electro-Optics, and Laser Applications in Science and Engineering (OE/LASE'93), 1866, Los Angeles, CA, USA, pp. 138-146.

Wong, N., Hall, J., 1985. Servo control of amplitude modulation in frequency-modulation spectroscopy: demonstration of shot-noise-limited detection. J. Opt. Soc. Am. B 2 (9), 1527-1533.

Ye, J., Ma, L.-S., Hall, J., 2001. Molecular iodine clock. Phys. Rev. Lett. 87 (27), 270801.

Zang, E., Cao, J., Li, Y., Li, C., Deng, Y., Gao, C., 2007. Realization of Four-Pass I2 Absorption Cell in 532-nm Optical Frequency Standard. IEEE Trans. Instrum. Measur. 56 (2), 673-676.

Zhang, W., Martin, M., Benko, C., Hall, J., Ye, J., Hagemann, C., et al., 2014. Reduction of residual amplitude modulation to $1 \mathrm{e}-6$ for frequency modulation and laser stabilization. Optics Lett. 39 (7), 1980-1983.

Zus, F., Douša, J., Kačmařík, M., Václavovic, P., Balidakis, K., Dick, G., Wickert, J., 2019a. Improving GNSS zenith wet delay interpolation by utilizing tropospheric gradients. Remote Sensing 11 (6), 674.

Zus, F., Douša, J., Kačmař́k, M., Václavovic, P., Dick, G., Wickert, J., 2019b. Estimating the impact of GNSS horizontal delay gradients in variational data assimilation. Remote Sensing 11 (1), 41. 